\title{
A MIXED CO-ROTATIONAL 3D BEAM ELEMENT FORMULATION FOR ARBITRARILY LARGE ROTATIONS
}

\author{
Z.X. $\mathrm{Li}^{1, *}$ and L. Vu-Quoc ${ }^{2}$ \\ ${ }^{1}$ Associate professor, Department of Civil Engineering, Zhejiang University, Hangzhou 310058, China \\ ${ }^{2}$ Professor, Department of Mechanical and Aerospace Engineering, University of Florida, \\ Gainesville FL 32611, USA \\ *(Corresponding author: E-mail: lizx19993@zju.edu.cn)
}

Received: 16 May 2009; Revised: 8 August 2009; Accepted: 11 August 2009

\begin{abstract}
A new 3-node co-rotational element formulation for 3D beam is presented. The present formulation differs from existing co-rotational formulations as follows: 1) vectorial rotational variables are used to replace traditional angular rotational variables, thus all nodal variables are additive in incremental solution procedure; 2) the Hellinger-Reissner functional is introduced to eliminate membrane and shear locking phenomena, with assumed membrane strains and shear strains employed to replace part of conforming strains; 3) all nodal variables are commutative in differentiating Hellinger-Reissner functional with respect to these variables, resulting in a symmetric element tangent stiffness matrix; 4) the total values of nodal variables are used to update the element tangent stiffness matrix, making it advantageous in solving dynamic problems. Several examples of elastic beams with large displacements and large rotations are analysed to verify the computational efficiency and reliability of the present beam element formulation.
\end{abstract}

Keywords: Co-rotational method; vectorial rotational variable; 3D beam element; locking-free; Hellinger-Reissner functional; assumed strain.

\section{INTRODUCTION}

Developing an efficient beam element formulation for large displacement analysis of framed structures has been an issue for many researchers. There already exist various formulations to address this issue. These formulations had been separated into three categories: Total Lagrangian formulation, updated Lagrangian formulation, and co-rotational formulation. The main ideas of the co-rotational approach (Rankin and Brogan [1], Crisfield [2], Yang et. al.[3]) can be summarized as follows: 1) define an element reference frame that translates and rotates with the element's overall rigid-body motion, but does not deform with the element; 2) calculate the nodal variables with respect to this reference frame; the element's overall rigid-body motion is thus excluded in computing the local internal force vector and the element tangent stiffness matrix, resulting an element-independent formulation; 3) the geometric nonlinearity induced by the large element rigid-body motion is incorporated in the transformation matrix relating the local and global internal force vector and tangent stiffness matrix.

Many co-rotational beam and shell element formulations have been proposed. The pioneer work can be traced to Wempner [4], Belytschko et al.[5,6], Argyris et al.[7] and Oran [8,9]. Surveys of the existing co-rotational finite element formulations were presented respectively by Stolarski et al.[10], Crisfield and Moita [11], Yang et al.[3], and Felippa and Haugen [12]. Recently, Urthaler and Reddy [13] developed three locking-free co-rotational planar beam element formulations by adopting respectively the Euler-Bernoulli, Timoshenko, and simplified Reddy theories in modelling of the element kinematic behaviour. Galvaneito and Crisfield [14] proposed an energy-conserving procedure for the implicit non-linear dynamic analysis of planar beam structures by using a form of co-rotational technique. Iura et al.[15] investigated the accuracy of the co-rotational formulation for 3-D Timoshenko beam undergoing finite strains and finite rotations. Pajot and Maute [16] studied the sensitivities of a co-rotational element formulation to element shape and material parameters, and the effect of the unsymmetric terms in a consistent tangent 
stiffness on element computational accuracy; see also Simo and Vu-Quoc [17] on the effect of tangent stiffness symmetrization on rate of convergence.

Due to the non-commutativity of spatial finite rotations, nodal rotations are always updated by using a complicated transformation matrix $[18,19]$ in an incremental solution procedure; such non-commutativity renders both the local and global element tangent stiffness matrices asymmetric in most existing co-rotational formulations. Thus more computer storage is needed to store all necessary coefficients, while the computational efficiency decreases. Simo and Vu-Quoc [17] proved that in a conservative system, although their tangent stiffness matrix is asymmetric away from equilibrium, this matrix becomes symmetric at equilibrium. Crisfield and his co-workers [2,20] also encountered this phenomenon, and artificially symmetrized the element tangent stiffness matrix by excluding the non-symmetric term. This treatment can greatly improve the computational efficiency. Crisfield [2] and Simo [21] also predicted that a symmetric tangent stiffness matrix could be achieved if a certain set of additive rotational variables were employed in a co-rotational element formulation. In the present co-rotational beam element formulation, such additive rotational variables are used, and the versatile vectorial rotational variables had also been employed in a co-rotational 2D beam element formulation [22], a co-rotational 3D beam conforming element formulation [23], a co-rotational curved triangular shell element formulation [24], and a co-rotational curved quadrilateral shell element formulation [25], respectively.

\section{DESCRIPTION OF THE CO-ROTATIONAL FRAMEWORK}

In the present beam element formulation, several basic assumptions were adopted: 1) the element is straight at the initial configuration; 2) the shape of the cross-section does not distort with element deforming; 3) the element cross-section is bisymmetric; 4) restrained warping effect is ignored.

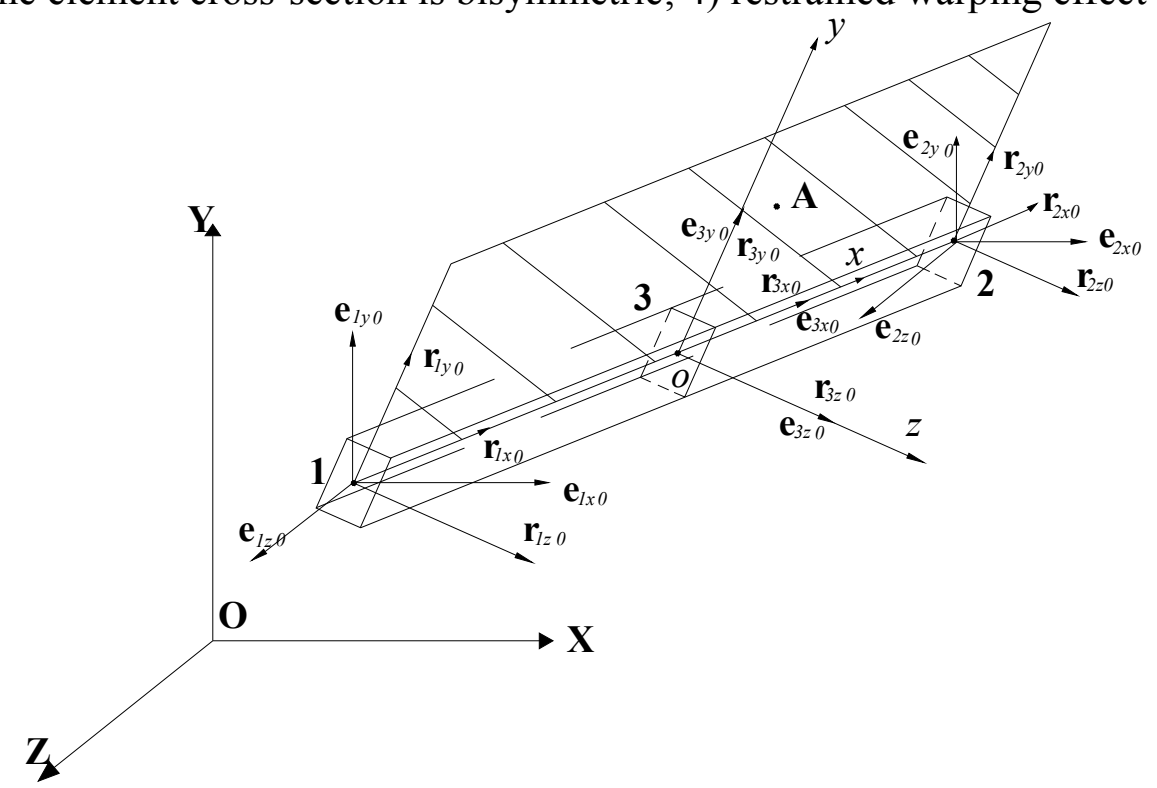

Figure 1. Definition of Local and Global Coordinate Systems

The local and global coordinate systems of the beam element are illustrated in Figure 1, where three local coordinate axes run along two principal axes of the cross-section at the internal node and their cross-product, and translate and rotate with the element rigid-body translations and rotations, but do not deform with the element. 
An auxiliary point in one of the symmetry plane of the beam element is employed in defining the local coordinate axes (see Point A in Figure 1). $\mathbf{e}_{x 0}, \mathbf{e}_{y 0}, \mathbf{e}_{z 0}$ are the normalized orientation vectors of $x$-axis, $y$-axis and $z$-axis, respectively. They are calculated from

$\left.\begin{array}{l}\mathbf{e}_{x 0}=\frac{\mathbf{v}_{120}}{\left|\mathbf{v}_{120}\right|} \\ \mathbf{e}_{z 0}=\frac{\mathbf{v}_{120} \times \mathbf{v}_{3 A 0} \mid}{\left|\mathbf{v}_{120} \times \mathbf{v}_{3 A 0}\right|} \\ \mathbf{e}_{y 0}=\mathbf{e}_{z 0} \times \mathbf{e}_{x 0}\end{array}\right\}$

where,

$\left.\begin{array}{l}\mathbf{v}_{120}=\mathbf{X}_{20}-\mathbf{X}_{10} \\ \mathbf{v}_{3 A 0}=\mathbf{X}_{A 0}-\mathbf{X}_{30}\end{array}\right\}$

and $\mathbf{X}_{i 0}(i=1,2,3, A)$ is the global coordinates of Node $i$.

The orientation vectors $\mathbf{e}_{i x}, \mathbf{e}_{i y}, \mathbf{e}_{i z}$ of Node $i$ at the deformed configuration are calculated from the rotational variables directly in an incremental solution procedure. In particular, at Node 3 (the internal node of the beam element), $\mathbf{e}_{3 x}, \mathbf{e}_{3 y}, \mathbf{e}_{3 z}$ are coincident with the orientation of local coordinate axes,

$\left.\begin{array}{rl}\mathbf{e}_{3 x} & =\mathbf{e}_{x} \\ \mathbf{e}_{3 y} & =\mathbf{e}_{y} \\ \mathbf{e}_{3 z} & =\mathbf{e}_{z}\end{array}\right\}$

and at the initial configuration,

$$
\left.\begin{array}{rl}
\mathbf{e}_{3 x 0} & =\mathbf{e}_{x 0} \\
\mathbf{e}_{3 y 0} & =\mathbf{e}_{y 0} \\
\mathbf{e}_{3 z 0} & =\mathbf{e}_{z 0}
\end{array}\right\}
$$

however, the initial orientation vectors of two end nodes are defined as

$$
\left.\begin{array}{l}
\mathbf{e}_{i x 0}^{\mathrm{T}}=\langle 1 \quad 00 \\
\mathbf{e}_{i y 0}^{\mathrm{T}}=\left\langle\begin{array}{lll}
0 & 1 & 0
\end{array}\right\rangle \\
\mathbf{e}_{i z 0}^{\mathrm{T}}=\left\langle\begin{array}{lll}
0 & 0 & 1
\end{array}\right\rangle
\end{array}\right\} \quad i=1,2
$$

In the global coordinate system, each element employs 18 degrees of freedom,

$$
\mathbf{u}_{\mathrm{G}}^{\mathrm{T}}=\left\langle\begin{array}{lllllllllllll}
U_{1} & V_{1} & W_{1} & e_{1 y, n_{1}} & e_{1 y, m_{1}} & e_{1 z, n_{1}} & \cdots & U_{3} & V_{3} & W_{3} & e_{3 y, n_{3}} & e_{3 y, m_{3}} & e_{3 z, n_{3}}
\end{array}\right\rangle
$$

where, $\mathbf{d}_{i}^{\mathrm{T}}=\left\langle\begin{array}{lll}U_{i} & V_{i} & W_{i}\end{array}\right\rangle$ is the vector of global translational displacements at Node $i$; 
$\mathbf{n}_{g i}^{\mathrm{T}}=\left\langle e_{i y, n_{i}} \quad e_{i y, m_{i}} \quad e_{i z, n_{i}}\right\rangle\left(n_{i}, m_{i}=X, Y\right.$ or $\left.Z\right)$ is the vector of vectorial rotational variables at Node $i$, it consists of three independent components of $\mathbf{e}_{i y}$ and $\mathbf{e}_{i z}$ in the global coordinate system.

In the local coordinate system, each element has 12 degrees of freedom, and each end node 6 freedoms,

$\mathbf{u}_{\mathrm{L}}^{\mathrm{T}}=\left\langle\begin{array}{llllllllllll}u_{1} & v_{1} & w_{1} & r_{1 y, n_{1}} & r_{1 y, m_{1}} & r_{1 z, n_{1}} & u_{2} & v_{2} & w_{2} & r_{2 y, n_{2}} & r_{2 y, m_{2}} & r_{2 z, n_{2}}\end{array}\right\rangle$

where, $\mathbf{t}_{i}^{\mathrm{T}}=\left\langle\begin{array}{lll}u_{i} & v_{i} & w_{i}\end{array}\right\rangle$ are the vector of local translational displacements at Node $i$, and $\boldsymbol{\theta}_{i}^{\mathrm{T}}=\left\langle r_{i y, n_{i}} r_{i y, m_{i}} \quad r_{i z, n_{i}}\right\rangle\left(n_{i}, m_{i}=x, y\right.$ or $\left.z\right)$ are the vector of vectorial rotational variables at Node $i$, it consists of three independent components of $\mathbf{e}_{i y}$ and $\mathbf{e}_{i z}$ in the local coordinate system.

The rotational variables $e_{i y, n_{i}}, e_{i y, m_{i}}$ and $e_{i z, n_{i}}$ are defined according to the following procedure.

Firstly, assumed that $\left|e_{i y, l_{i}}\right| \geq\left|e_{i y, m_{i}}\right|,\left|e_{i y, l_{i}}\right| \geq\left|e_{i y, n_{i}}\right| \quad\left(l_{i}, m_{i}, n_{i} \in\{X, Y, Z\}\right.$, and $\left.l_{i} \neq m_{i} \neq n_{i}\right)$ at the preceding incremental loading step:

Case 1: if $\left|e_{i z, l_{i}}\right| \geq\left|e_{i z, m_{i}}\right|$ and $\left|e_{i z, l_{i}}\right| \geq\left|e_{i z, n_{i}}\right|$, then three rotational variables at the next incremental loading step are $\mathbf{n}_{g i}^{\mathrm{T}}=\left\langle e_{i y, n_{i}} e_{i y, m_{i}} e_{i z, n_{i}}\right\rangle$, where $\left\{n_{i}, m_{i} l_{i}\right\}$ is a circular permutation of $\{X, Y, Z\}$, other components of $\mathbf{e}_{i y}$ and $\mathbf{e}_{i z}$ can be calculated from them,

$$
\begin{aligned}
& e_{i y, l_{i}}=s_{1} \sqrt{1-e_{i y, n_{i}}^{2}-e_{i y, m_{i}}^{2}} \\
& e_{i z, m_{i}}=\frac{-e_{i y, m_{i}} e_{i y, n_{i}} e_{i z, n_{i}}+s_{2} e_{i y, l_{i}} \sqrt{1-e_{i y, n_{i}}^{2}-e_{i z, n_{i}}^{2}}}{1-e_{i y, n_{i}}^{2}} \\
& e_{i z, l_{i}}=s_{3} \sqrt{1-e_{i z, m_{i}}^{2}-e_{i z, n_{i}}^{2}}
\end{aligned}
$$

where, $s_{1}, s_{3}$ take a numeric value of 1 or -1 , they have the same signs as $e_{i y, l_{i}}$ or $e_{i z, l_{i}}$ at last incremental step; $s_{2}$ is also such a constant, and it is conditioned on $\mathbf{e}_{i y}^{\mathrm{T}} \mathbf{e}_{i z}=0$.

Case 2: if $\left|e_{i z, m_{i}}\right| \geq\left|e_{i z, l_{i}}\right|$ and $\left|e_{i z, m_{i}}\right| \geq\left|e_{i z, n_{i}}\right|$ at the end of the current incremental step, then three rotational variables are defined as $\mathbf{n}_{g i}^{\mathrm{T}}=\left\langle\begin{array}{lll}e_{i y, n_{i}} & e_{i y, m_{i}} & e_{i z, n_{i}}\end{array}\right\rangle$, and other components of $\mathbf{e}_{i y}$ and $\mathbf{e}_{i z}$ can be calculated as,

$$
\begin{aligned}
& e_{i y, l_{i}}=s_{1} \sqrt{1-e_{i y, n_{i}}^{2}-e_{i y, m_{i}}^{2}} \\
& e_{i z, l_{i}}=\frac{-s_{1} \sqrt{1-e_{i y, m_{i}}^{2}-e_{i y, n_{i}}^{2}} e_{i y, n_{i}} e_{i z, n_{i}}+s_{2} e_{i y, m_{i}} \sqrt{1-e_{i y, n_{i}}^{2}-e_{i z, n_{i}}^{2}}}{1-e_{i y, n_{i}}^{2}} \\
& e_{i z, m_{i}}=s_{3} \sqrt{1-e_{i z, n_{i}}^{2}-e_{i z, l_{i}}^{2}}
\end{aligned}
$$


where, $s_{1}, s_{2}, s_{3}$ are the same kind of constants as those in Case 1.

Vector $\mathbf{e}_{i x}$ is the cross-product of Vectors $\mathbf{e}_{i y}$ and $\mathbf{e}_{i z}$,

$\mathbf{e}_{i x}=\mathbf{e}_{i y} \times \mathbf{e}_{i z}$

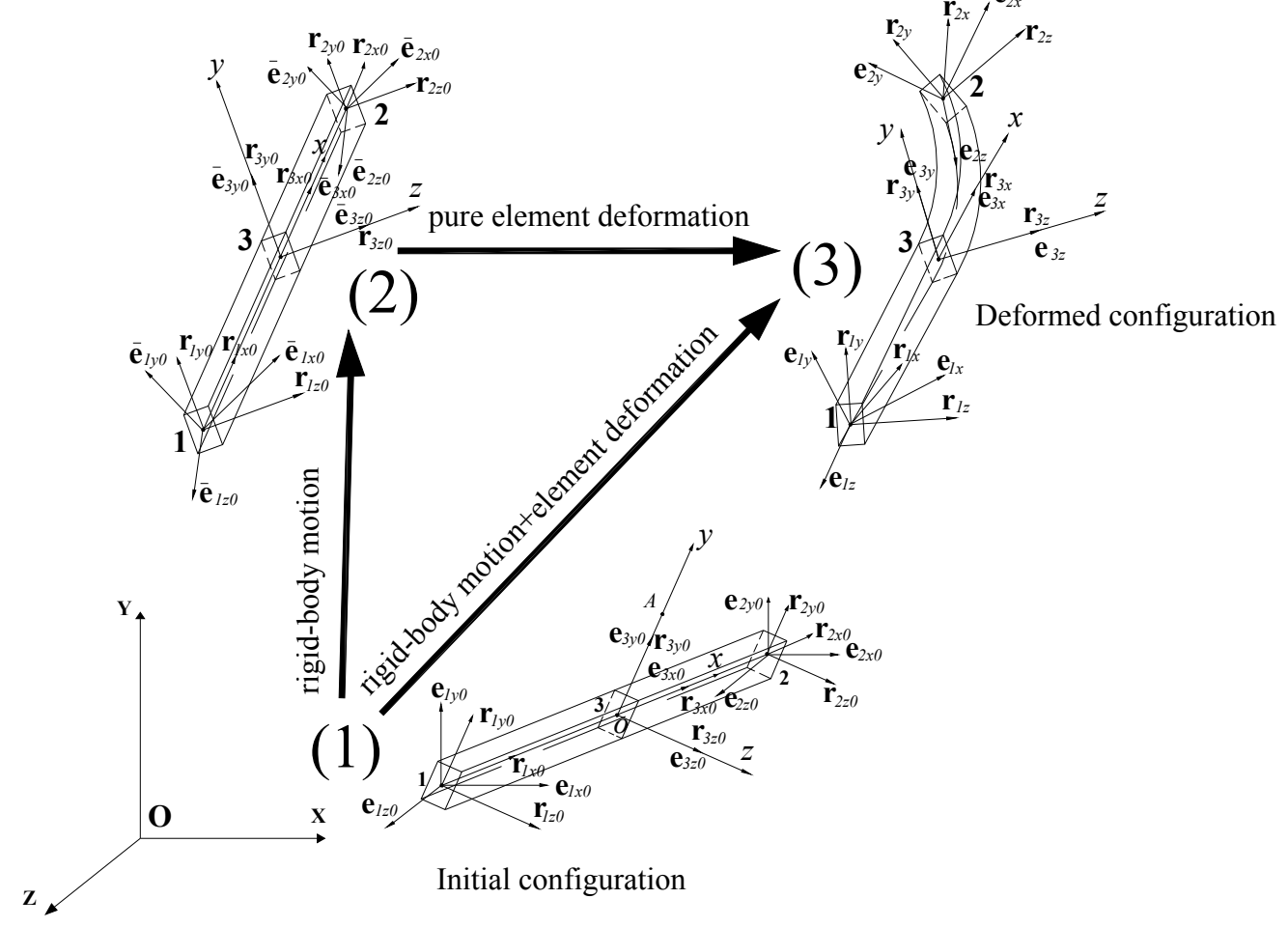

Figure 2. Illumination of the Co-rotational Framework

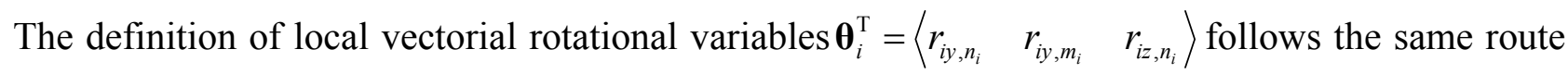
as that of $\mathbf{n}_{g i}^{\mathrm{T}}=\left\langle\begin{array}{lll}e_{i y, n_{i}} & e_{i y, m_{i}} & e_{i z, n_{i}}\end{array}\right\rangle$.

Rigid-body motion contributes nothing to element strains, so it can be excluded in advance to achieve an element-independent formulation. This procedure is illuminated in Figure 2, where (1) represents an element at its initial configuration, (3) is at the deformed configuration, from (1) to (3) the element experiences both rigid-body motion and pure deformation. (2) is an intermediate configuration between (1) and (3), from (1) to (2), the element has only rigid-body motion, while, from (2) to (3), the element experiences pure deformation. In the present co-rotational formulation, the rigid-body motion from (1) to (2) is excluded, and only pure element deformation from (2) to (3) is considered in calculating the local internal force vector and element tangent stiffness matrix. Thus, the relationships between local and global nodal variables are given as,

$\left.\begin{array}{l}\mathbf{t}_{i}=\mathbf{R}\left(\mathbf{d}_{i}-\mathbf{d}_{3}\right)+\left(\mathbf{R}-\mathbf{R}_{0}\right) \mathbf{v}_{i 0} \\ \mathbf{r}_{i y}=\mathbf{R} \mathbf{R}_{i}^{\mathrm{T}} \mathbf{e}_{y 0} \\ \mathbf{r}_{i z}=\mathbf{R} \mathbf{R}_{i}^{\mathrm{T}} \mathbf{e}_{z 0}\end{array}\right\} \quad i=1,2$

where, 


$$
\begin{aligned}
& \mathbf{R}_{0}=\left[\begin{array}{c}
\mathbf{e}_{x 0}^{\mathrm{T}} \\
\mathbf{e}_{y 0}^{\mathrm{T}} \\
\mathbf{e}_{z 0}^{\mathrm{T}}
\end{array}\right] \\
& \mathbf{R}=\left[\begin{array}{c}
\mathbf{e}_{x}^{\mathrm{T}} \\
\mathbf{e}_{y}^{\mathrm{T}} \\
\mathbf{e}_{z}^{\mathrm{T}}
\end{array}\right] \\
& \mathbf{R}_{i}=\left[\begin{array}{c}
\mathbf{e}_{i x}^{\mathrm{T}} \\
\mathbf{e}_{i y}^{\mathrm{T}} \\
\mathbf{e}_{i z}^{\mathrm{T}}
\end{array}\right] \quad i=1,2
\end{aligned}
$$

Especially, at the internal node,

$$
\left.\begin{array}{l}
\mathbf{t}_{3}^{\mathrm{T}}=\left\langle\begin{array}{lll}
0 & 0 & 0
\end{array}\right\rangle \\
\mathbf{r}_{3 y}^{\mathrm{T}}=\left\langle\begin{array}{lll}
0 & 1 & 0
\end{array}\right\rangle \\
\mathbf{r}_{3 z}^{\mathrm{T}}=\left\langle\begin{array}{lll}
0 & 0 & 1
\end{array}\right\rangle
\end{array}\right\}
$$

$\mathbf{v}_{i 0}$ is the relative vector oriented from Node 3 to Node $i$,

$$
\mathbf{v}_{i 0}=\mathbf{X}_{i 0}-\mathbf{X}_{30} \quad i=1,2
$$

\section{KINEMATICS OF A 3-NODE ISO-PARAMETRIC BEAM ELEMENT}

In the present 3-node iso-parametric beam element, the local coordinates, displacements and vectorial rotations at any point of the element central line are interpolated by using Lagrangian shape functions. The initial and current local coordinates at any point of the element can be depicted as

$$
\begin{aligned}
& { }^{0} \mathbf{g}=\sum_{i=1}^{3} N_{i}(\xi) \mathbf{x}_{i 0}+y_{l} \sum_{i=1}^{3} N_{i}(\xi) \mathbf{r}_{i j 0}+z_{l} \sum_{i=1}^{3} N_{i}(\xi) \mathbf{r}_{i z 0} \\
& \mathbf{g}=\sum_{i=1}^{3} N_{i}(\xi)\left(\mathbf{t}_{i}+\mathbf{x}_{i 0}\right)+y_{l} \sum_{i=1}^{3} N_{i}(\xi) \mathbf{r}_{i j}+z_{l} \sum_{i=1}^{3} N_{i}(\xi) \mathbf{r}_{i z}
\end{aligned}
$$

where, $N_{i}(\xi)$ is the Lagrangian shape function at Node $i ; \xi$ is the natural coordinate of a point in the element along its central line; $\mathbf{x}_{i 0}$ is the initial local coordinates of Node $i ; y_{l}$ and $z_{l}$ are the relative coordinates of any point in the element to its central line.

Considering the possibility of large displacements and large rotations, Green strain measure is introduced to describe the strain-displacement relationship. For a beam element, the strain-displacement relationship is given as 
$\boldsymbol{\varepsilon}=\left\{\begin{array}{l}\varepsilon_{x x} \\ \gamma_{x y} \\ \gamma_{x z}\end{array}\right\}=\left\{\begin{array}{c}\frac{1}{2}\left(\mathbf{g}_{x}^{\mathrm{T}} \mathbf{g}_{x}-{ }^{0} \mathbf{g}_{x}^{\mathrm{T} 0} \mathbf{g}_{x}\right) \\ \mathbf{g}_{x}^{\mathrm{T}} \mathbf{g}_{y}-{ }^{0} \mathbf{g}_{x}^{\mathrm{T} 0} \mathbf{g}_{y} \\ \mathbf{g}_{x}^{\mathrm{T}} \mathbf{g}_{z}-{ }^{0} \mathbf{g}_{x}^{\mathrm{T} 0} \mathbf{g}_{z}\end{array}\right\}$

where,

$$
\left.\begin{array}{c}
{ }^{0} \mathbf{g}_{x}=\frac{\partial^{0} \mathbf{g}}{\partial x} \\
\mathbf{g}_{x}=\frac{\partial \mathbf{g}}{\partial x}
\end{array}\right\}
$$

For convenience, Eq. 16 can be rewritten as

$$
\boldsymbol{\varepsilon}=\boldsymbol{\varepsilon}^{(0)}+y_{l} \boldsymbol{\varepsilon}^{(1)}+z_{l} \boldsymbol{\varepsilon}^{(2)}+y_{l} z_{l} \boldsymbol{\varepsilon}^{(3)}+y_{l}^{2} \boldsymbol{\varepsilon}^{(4)}+z_{l}^{2} \boldsymbol{\varepsilon}^{(5)}
$$

where,

$$
\begin{aligned}
& \boldsymbol{\varepsilon}^{(0)}=\left\{\begin{array}{l}
\varepsilon_{x x}^{(0)} \\
\gamma_{x y}^{(0)} \\
\gamma_{x z}^{(0)}
\end{array}\right\}=\left\{\begin{array}{c}
\frac{1}{2} \frac{\partial \mathbf{u}_{0}^{\mathrm{T}}}{\partial x} \frac{\partial \mathbf{u}_{0}}{\partial x}+\frac{\partial \mathbf{u}_{0}^{\mathrm{T}}}{\partial x} \frac{\partial \mathbf{x}}{\partial x} \\
\frac{\partial \mathbf{u}_{0}^{\mathrm{T}}}{\partial x} \mathbf{r}_{y}+\frac{\partial \mathbf{x}^{\mathrm{T}}}{\partial x}\left(\mathbf{r}_{y}-\mathbf{r}_{y 0}\right) \\
\frac{\partial \mathbf{u}_{0}^{\mathrm{T}}}{\partial x} \mathbf{r}_{z}+\frac{\partial \mathbf{x}^{\mathrm{T}}}{\partial x}\left(\mathbf{r}_{z}-\mathbf{r}_{z 0}\right)
\end{array}\right\} \\
& \boldsymbol{\varepsilon}^{(1)}=\left\{\begin{array}{l}
\varepsilon_{x x}^{(1)} \\
\gamma_{x y}^{(1)} \\
\gamma_{x z}^{(1)}
\end{array}\right\}=\left\{\begin{array}{c}
\frac{\partial \mathbf{u}_{0}^{\mathrm{T}}}{\partial x} \frac{\partial \mathbf{r}_{y}}{\partial x}+\frac{\partial \mathbf{x}^{\mathrm{T}}}{\partial x} \frac{\partial\left(\mathbf{r}_{y}-\mathbf{r}_{y 0}\right)}{\partial x} \\
\frac{\partial \mathbf{r}_{y}^{\mathrm{T}}}{\partial x} \mathbf{r}_{y}-\frac{\partial \mathbf{r}_{y 0}^{\mathrm{T}}}{\partial x} \mathbf{r}_{y 0} \\
\frac{\partial \mathbf{r}_{y}^{\mathrm{T}}}{\partial x} \mathbf{r}_{z}-\frac{\partial \mathbf{r}_{y 0}^{\mathrm{T}}}{\partial x} \mathbf{r}_{z 0}
\end{array}\right\} \\
& \boldsymbol{\varepsilon}^{(2)}=\left\{\begin{array}{l}
\varepsilon_{x x}^{(2)} \\
\gamma_{x y}^{(2)} \\
\gamma_{x z}^{(2)}
\end{array}\right\}=\left\{\begin{array}{c}
\frac{\partial \mathbf{u}_{0}^{\mathrm{T}}}{\partial x} \frac{\partial \mathbf{r}_{z}}{\partial x}+\frac{\partial \mathbf{x}^{\mathrm{T}}}{\partial x} \frac{\partial\left(\mathbf{r}_{z}-\mathbf{r}_{z 0}\right)}{\partial x} \\
\frac{\partial \mathbf{r}_{z}^{\mathrm{T}}}{\partial x} \mathbf{r}_{y}-\frac{\partial \mathbf{r}_{z 0}^{\mathrm{T}}}{\partial x} \mathbf{r}_{y 0} \\
\frac{\partial \mathbf{r}_{z}^{\mathrm{T}}}{\partial x} \mathbf{r}_{z}-\frac{\partial \mathbf{r}_{z 0}^{\mathrm{T}}}{\partial x} \mathbf{r}_{z 0}
\end{array}\right\} \\
& \boldsymbol{\varepsilon}^{(3)}=\left\{\begin{array}{l}
\varepsilon_{x x}^{(3)} \\
\gamma_{x y}^{(3)} \\
\gamma_{x z}^{(3)}
\end{array}\right\}=\left\{\begin{array}{c}
\frac{\partial \mathbf{r}_{y}^{\mathrm{T}}}{\partial x} \frac{\partial \mathbf{r}_{z}}{\partial x}-\frac{\partial \mathbf{r}_{y 0}^{\mathrm{T}}}{\partial x} \frac{\partial \mathbf{r}_{z 0}}{\partial x} \\
0 \\
0
\end{array}\right\}
\end{aligned}
$$


$\boldsymbol{\varepsilon}^{(4)}=\left\{\begin{array}{l}\varepsilon_{x x}^{(4)} \\ \gamma_{x y}^{(4)} \\ \gamma_{x z}^{(4)}\end{array}\right\}=\left\{\begin{array}{c}\frac{1}{2}\left(\frac{\partial \mathbf{r}_{y}}{\partial x} \frac{\partial \mathbf{r}_{y}}{\partial x}-\frac{\partial \mathbf{r}_{y 0}}{\partial x} \frac{\partial \mathbf{r}_{y 0}}{\partial x}\right) \\ 0 \\ 0\end{array}\right\}$
$\boldsymbol{\varepsilon}^{(5)}=\left\{\begin{array}{c}\varepsilon_{x x}^{(5)} \\ \gamma_{x y}^{(5)} \\ \gamma_{x z}^{(5)}\end{array}\right\}=\left\{\begin{array}{c}\frac{1}{2}\left(\frac{\partial \mathbf{r}_{z}^{\mathrm{T}}}{\partial x} \frac{\partial \mathbf{r}_{z}}{\partial x}-\frac{\partial \mathbf{r}_{z 0}^{\mathrm{T}}}{\partial x} \frac{\partial \mathbf{r}_{z 0}}{\partial x}\right) \\ 0 \\ 0\end{array}\right\}$

in Eqs. 19a f,

$\mathbf{u}_{0}=\sum_{i=1}^{3} N_{i}(\xi) \mathbf{t}_{i}$

$\mathbf{r}_{y}=\sum_{i=1}^{3} N_{i}(\xi) \mathbf{r}_{i y}$

$\mathbf{r}_{y 0}=\sum_{i=1}^{3} N_{i}(\xi) \mathbf{r}_{i y 0}$

$\mathbf{r}_{z}=\sum_{i=1}^{3} N_{i}(\xi) \mathbf{r}_{i z}$

$\mathbf{r}_{z 0}=\sum_{i=1}^{3} N_{i}(\xi) \mathbf{r}_{i z 0}$

$\frac{\partial}{\partial x}=\frac{\partial}{\partial \xi} / \sum_{i=1}^{3}\left(N_{i, \xi} x_{i 0}\right)$

in Eq. 20f, $N_{i, \xi}$ represents the first derivative of $N_{i}(\xi)$ with respect to $\xi$.

\section{ELEMENT FORMULATION}

To eliminate membrane and shear locking phenomena in beam elements, Hellinger-Reissner mixed functional are employed, where part of conforming strains are replaced by assumed strains,

$$
\begin{aligned}
& \pi_{\mathrm{HR}}=\mathrm{E} \int_{\mathrm{V}} \varepsilon_{x x}^{a} \varepsilon_{x x} \mathrm{dV}-\frac{1}{2} \mathrm{E} \int_{\mathrm{V}}\left(\varepsilon_{x x}^{a}\right)^{2} \mathrm{dV}+\mathrm{k}_{0} \mathrm{G} \int_{\mathrm{V}} \gamma_{x y}^{a} \gamma_{x y} \mathrm{dV} \\
& -\frac{1}{2} \mathrm{k}_{0} \mathrm{G} \int_{\mathrm{V}}\left(\gamma_{x y}^{a}\right)^{2} \mathrm{dV}+\mathrm{k}_{0} \mathrm{G} \int_{\mathrm{V}} \gamma_{x z}^{a} \gamma_{x z} \mathrm{dV}-\frac{1}{2} \mathrm{k}_{0} \mathrm{G} \int_{\mathrm{V}}\left(\gamma_{x z}^{a}\right)^{2} \mathrm{dV}-\mathrm{W}_{e}
\end{aligned}
$$


where, $\mathrm{E}$ and $\mathrm{G}$ are the Young's modulus and the shear modulus, respectively; $\mathrm{k}_{0}$ is the shear factor of the cross-section; $\mathrm{V}$ is the element volume; $\mathrm{W}_{e}$ is the work done by external forces; and

$$
\begin{aligned}
& \varepsilon_{x x}^{a}=\mathbf{P} \boldsymbol{\alpha}+y_{l} \varepsilon_{x x}^{(1)}+z_{l} \varepsilon_{x x}^{(2)}+y_{l} z_{l} \varepsilon_{x x}^{(3)}+y_{l}^{2} \varepsilon_{x x}^{(4)}+z_{l}^{2} \varepsilon_{x x}^{(5)} \\
& \gamma_{x y}^{a}=\mathbf{P} \boldsymbol{\beta}+y_{l} \gamma_{x y}^{(1)}+z_{l} \gamma_{x y}^{(2)} \\
& \gamma_{x z}^{a}=\mathbf{P} \chi+y_{l} \gamma_{x z}^{(1)}+z_{l} \gamma_{x z}^{(2)} \\
& \mathbf{P}=\langle 1, \xi\rangle \\
& \boldsymbol{\alpha}=\left\langle\alpha_{1}, \alpha_{2}\right\rangle^{\mathrm{T}} \\
& \boldsymbol{\beta}=\left\langle\beta_{1}, \beta_{2}\right\rangle^{\mathrm{T}} \\
& \chi=\left\langle\chi_{1}, \chi_{2}\right\rangle^{\mathrm{T}}
\end{aligned}
$$

$\alpha_{1}, \alpha_{2}, \beta_{1}, \beta_{2}, \chi_{1}, \chi_{2}$ are independent variables employed in defining assumed strains.

By enforcing the variation of the Hellinger-Reissner functional $\pi_{\mathrm{HR}}$ with respect to $\mathbf{u}_{\mathbf{L}}, \boldsymbol{\alpha}, \boldsymbol{\beta}$ and $\chi$,

$$
\begin{aligned}
\delta \pi_{\mathrm{HR}}= & \mathrm{E} \int_{\mathrm{V}} \varepsilon_{x x}^{a} \delta \varepsilon_{x x} \mathrm{dV}+\mathrm{E} \int_{\mathrm{V}} \varepsilon_{x x} \delta \varepsilon_{x x}^{a} \mathrm{dV}-\mathrm{E} \int_{\mathrm{V}} \varepsilon_{x x}^{a} \delta \varepsilon_{x x}^{a} \mathrm{dV} \\
& +\mathrm{k}_{0} \mathrm{G} \int_{\mathrm{V}} \gamma_{x y}^{a} \delta \gamma_{x y} \mathrm{dV}+\mathrm{k}_{0} \mathrm{G} \int_{\mathrm{V}} \gamma_{x y} \delta \gamma_{x y}^{a} \mathrm{dV} \\
& -\mathrm{k}_{0} \mathrm{G} \int_{\mathrm{V}} \gamma_{x y}^{a} \delta \gamma_{x y}^{a} \mathrm{dV}+\mathrm{k}_{0} \mathrm{G} \int_{\mathrm{V}} \gamma_{x z}^{a} \delta \gamma_{x z} d \mathrm{~V} \\
& +\mathrm{k}_{0} \mathrm{G} \int_{\mathrm{V}} \gamma_{x z} \delta \gamma_{x z}^{a} \mathrm{dV}-\mathrm{k}_{0} \mathrm{G} \int_{\mathrm{V}} \gamma_{x z}^{a} \delta \gamma_{x z}^{a} \mathrm{dV}-\delta \mathrm{W}_{e}
\end{aligned}
$$

where,

$$
\begin{aligned}
& \delta \varepsilon_{x x}^{a}=\mathbf{P} \delta \boldsymbol{a}+\left(y_{l} \mathbf{B}_{x x}^{(1)}+z_{l} \mathbf{B}_{x x}^{(2)}+y_{l} z_{l} \mathbf{B}_{x x}^{(3)}+y_{l}^{2} \mathbf{B}_{x x}^{(4)}+z_{l}^{2} \mathbf{B}_{x x}^{(5)}\right) \delta \mathbf{u}_{\mathrm{L}} \\
& \delta \varepsilon_{x x}=\left(\mathbf{B}_{x x}^{(0)}+y_{l} \mathbf{B}_{x x}^{(1)}+z_{l} \mathbf{B}_{x x}^{(2)}+y_{l} z_{l} \mathbf{B}_{x x}^{(3)}+y_{l}^{2} \mathbf{B}_{x x}^{(4)}+z_{l}^{2} \mathbf{B}_{x x}^{(5)}\right) \delta \mathbf{u}_{\mathbf{L}} \\
& \delta \gamma_{x y}^{a}=\mathbf{P} \delta \boldsymbol{\beta}+\left(y_{l} \mathbf{B}_{x y}^{(1)}+z_{l} \mathbf{B}_{x y}^{(2)}\right) \delta \mathbf{u}_{\mathrm{L}} \\
& \delta \gamma_{x y}=\left(\mathbf{B}_{x y}^{(0)}+y_{l} \mathbf{B}_{x y}^{(1)}+z_{l} \mathbf{B}_{x y}^{(2)}\right) \delta \mathbf{u}_{\mathrm{L}} \\
& \delta \gamma_{x z}^{a}=\mathbf{P} \delta \boldsymbol{\chi}+\left(y_{l} \mathbf{B}_{x z}^{(1)}+z_{l} \mathbf{B}_{x z}^{(2)}\right) \delta \mathbf{u}_{\mathrm{L}} \\
& \delta \gamma_{x z}=\left(\mathbf{B}_{x z}^{(0)}+y_{l} \mathbf{B}_{x z}^{(1)}+z_{l} \mathbf{B}_{x z}^{(2)}\right) \delta \mathbf{u}_{\mathrm{L}} \\
& \delta \mathrm{W}_{e}=\mathbf{f}_{\mathrm{ext}}^{\mathrm{T}} \delta \mathbf{u}_{\mathrm{L}}
\end{aligned}
$$


considering the independence of $\delta \boldsymbol{\alpha}, \delta \boldsymbol{\beta}, \delta \boldsymbol{\chi}$ and $\delta \mathbf{u}_{\mathrm{L}}, \mathbf{f}=\mathbf{f}_{\text {ext }}$ at the equilibrium state, and assumed that the cross-section of the beam element is bisymmetric, meanwhile, let

$$
\begin{aligned}
& \mathbf{H}=\mathrm{A} \int_{\mathrm{L}} \mathbf{P}^{\mathrm{T}} \mathbf{P} \mathrm{d} x \\
& \mathbf{F}_{1}=\mathrm{A} \int_{\mathrm{L}} \mathbf{P}^{\mathrm{T}} \varepsilon_{x x}^{(0)} \mathrm{d} x \\
& \mathbf{F}_{2}=\mathrm{A} \int_{\mathrm{L}} \mathbf{P}^{\mathrm{T}} \gamma_{x y}^{(0)} \mathrm{d} x \\
& \mathbf{F}_{3}=\mathrm{A} \int_{\mathrm{L}} \mathbf{P}^{\mathrm{T}} \gamma_{x z}^{(0)} \mathrm{d} x
\end{aligned}
$$

then,

$$
\boldsymbol{\alpha}=\mathbf{H}^{-1} \mathbf{F}_{1}
$$

$\boldsymbol{\beta}=\mathbf{H}^{-1} \mathbf{F}_{2}$

$$
\chi=\mathbf{H}^{-1} \mathbf{F}_{3}
$$

and the local internal force vector $\mathbf{f}$ of the element can be calculated by

$$
\begin{aligned}
& \mathbf{f}=\mathrm{E} \int_{\mathrm{L}}\left[\mathrm{I} y\left(\mathbf{B}_{x x}^{(0)^{\mathrm{T}}}{ }^{(5)}+\mathbf{B}_{x x}^{(2)^{\mathrm{T}}} \varepsilon_{x x}^{(2)}+\mathbf{B}_{x x}^{(5)^{\mathrm{T}}} \varepsilon_{x x}^{(0)}\right)+\mathrm{I}_{\omega y} \mathbf{B}_{x x}^{(5)^{\mathrm{T}}} \varepsilon_{x x}^{(5)}\right] \mathrm{d} x+\mathrm{E} A \int_{\mathrm{L}} \mathbf{B}_{x x}^{(0)^{\mathrm{T}}} \mathbf{P} \mathrm{d} x \boldsymbol{\alpha} \\
& +\mathrm{E} \int_{\mathrm{L}}\left[\mathrm{I}_{z}\left(\mathbf{B}_{x x}^{(0)^{\mathrm{T}}} \varepsilon_{x x}^{(4)}+\mathbf{B}_{x x}^{(1)^{\mathrm{T}}} \varepsilon_{x x}^{(1)}+\mathbf{B}_{x x}^{(4)^{\mathrm{T}}} \varepsilon_{x x}^{(0)}\right)+\mathrm{I}_{\omega z} \mathbf{B}_{x x}^{(4)^{\mathrm{T}}} \varepsilon_{x x}^{(4)}\right] \mathrm{d} x \\
& +\mathrm{EI}_{\omega y z} \int_{\mathrm{L}}\left(\mathbf{B}_{x x}^{(4)^{\mathrm{T}}} \varepsilon_{x x}^{(5)}+\mathbf{B}_{x x}^{(5)^{\mathrm{T}}} \varepsilon_{x x}^{(4)}+\mathbf{B}_{x x}^{(3)^{\mathrm{T}}} \varepsilon_{x x}^{(3)}\right) \mathrm{d} x \\
& +\mathrm{k}_{0} \mathrm{G}\left[\iint_{\mathrm{L}}\left(\mathrm{I}_{y} \mathbf{B}_{x y}^{(2)}{ }^{\mathrm{T}} \gamma_{x y}^{(2)}+\mathrm{I}_{z} \mathbf{B}_{x y}^{(1)^{\mathrm{T}}} \gamma_{x y}^{(1)}\right) \mathrm{d} x+\mathrm{A} \int_{\mathrm{L}} \mathbf{B}_{x y}^{(0)}{ }^{\mathrm{T}} \mathbf{P} \mathrm{d} x \boldsymbol{\beta}\right] \\
& +\mathrm{k}_{0} \mathrm{G}\left[\iint_{\mathrm{L}}\left(\mathrm{I}_{y} \mathbf{B}_{x z}^{(2)^{\mathrm{T}}} \gamma_{x z}^{(2)}+\mathrm{I}_{z} \mathbf{B}_{x z}^{(1)^{\mathrm{T}}} \gamma_{x z}^{(1)}\right) \mathrm{d} x+\mathrm{A} \int_{\mathrm{L}} \mathbf{B}_{x z}^{(0)^{\mathrm{T}}} \mathbf{P} \mathrm{d} x \boldsymbol{\chi}\right]
\end{aligned}
$$

where, $\mathrm{A}$ is the cross-sectional area of the element, $\mathrm{I}_{y}=\int_{\mathrm{A}} z_{l}^{2} \mathrm{dA}, \mathrm{I}_{z}=\int_{\mathrm{A}} y_{l}^{2} \mathrm{dA}, \mathrm{I}_{\omega y}=\int_{\mathrm{A}} z_{l}^{4} \mathrm{dA}$, $I_{\omega z}=\int_{\mathrm{A}} y_{l}^{4} \mathrm{dA}, \mathrm{I}_{\omega y z}=\int_{\mathrm{A}} y_{l}^{2} z_{l}^{2} \mathrm{dA}$

The element tangent stiffness matrix in the local coordinate system can be calculated from differentiating the local internal force vector of the element with respect to $\mathbf{u}_{\mathrm{L}}$, 


$$
\begin{aligned}
& \mathbf{k}_{\mathrm{T}}=\mathrm{E} \int_{\mathrm{L}}\left[\mathrm{I}_{y}\left(\mathbf{B}_{x x}^{(2)}{ }^{\mathrm{T}} \mathbf{B}_{x x}^{(2)}+\mathbf{B}_{x x}^{(5)}{ }^{\mathrm{T}} \mathbf{B}_{x x}^{(0)}+\mathbf{B}_{x x}^{(0)}{ }^{\mathrm{T}} \mathbf{B}_{x x}^{(5)}\right)+\mathrm{I}_{\omega y} \mathbf{B}_{x x}^{(5)}{ }^{\mathrm{T}} \mathbf{B}_{x x}^{(5)}\right] \mathrm{d} x \\
& +\mathrm{E} \int_{\mathrm{L}}\left[\mathrm{I}_{z}\left(\mathbf{B}_{x x}^{(1)^{\mathrm{T}}} \mathbf{B}_{x x}^{(1)}+\mathbf{B}_{x x}^{(4)^{\mathrm{T}}} \mathbf{B}_{x x}^{(0)}+\mathbf{B}_{x x}^{(0)^{\mathrm{T}}} \mathbf{B}_{x x}^{(4)}\right)+\mathrm{I}_{\omega z} \mathbf{B}_{x x}^{(4)}{ }^{\mathrm{T}} \mathbf{B}_{x x}^{(4)}\right] \mathrm{d} x \\
& +\mathrm{I}_{\omega y z} \mathrm{E} \int_{\mathrm{L}}\left(\mathbf{B}_{x x}^{(3)}{ }^{\mathrm{T}} \mathbf{B}_{x x}^{(3)}+\mathbf{B}_{x x}^{(4)} \mathbf{B}_{x x}^{\mathrm{T}}+\mathbf{B}_{x x}^{(5)}{ }^{\mathrm{T}} \mathbf{B}_{x x}^{(4)}\right) \mathrm{d} x \\
& +\mathrm{E} \int_{\mathrm{L}}\left[\mathrm{I}_{y}\left(\frac{\partial \mathbf{B}_{x x}^{(2)^{\mathrm{T}}}}{\partial \mathbf{u}_{\mathrm{L}}^{\mathrm{T}}} \varepsilon_{x x}^{(2)}+\frac{\partial \mathbf{B}_{x x}^{(5)^{\mathrm{T}}}}{\partial \mathbf{u}_{\mathrm{L}}^{\mathrm{T}}} \varepsilon_{x x}^{(0)}+\frac{\partial \mathbf{B}_{x x}^{(0)^{\mathrm{T}}}}{\partial \mathbf{u}_{\mathrm{L}}^{\mathrm{T}}} \varepsilon_{x x}^{(5)}\right)+\mathrm{I}_{\omega y} \frac{\partial \mathbf{B}_{x x}^{(5)^{\mathrm{T}}}}{\partial \mathbf{u}_{\mathrm{L}}^{\mathrm{T}}} \varepsilon_{x x}^{(5)}\right] \mathrm{d} x \\
& +\mathrm{E} \int_{\mathrm{L}}\left[\mathrm{I}_{z}\left(\frac{\partial \mathbf{B}_{x x}^{(1)^{\mathrm{T}}}}{\partial \mathbf{u}_{\mathrm{L}}^{\mathrm{T}}} \varepsilon_{x x}^{(1)}+\frac{\partial \mathbf{B}_{x x}^{(4)^{\mathrm{T}}}}{\partial \mathbf{u}_{\mathrm{L}}^{\mathrm{T}}} \varepsilon_{x x}^{(0)}+\frac{\partial \mathbf{B}_{x x}^{(0)^{\mathrm{T}}}}{\partial \mathbf{u}_{\mathrm{L}}^{\mathrm{T}}} \varepsilon_{x x}^{(4)}\right)+\mathrm{I}_{\omega z} \frac{\partial \mathbf{B}_{x x}^{(4)^{\mathrm{T}}}}{\partial \mathbf{u}_{\mathrm{L}}^{\mathrm{T}}} \varepsilon_{x x}^{(4)}\right] \mathrm{d} x \\
& +\mathrm{EI}_{\omega y z} \int_{\mathrm{L}}\left(\frac{\partial \mathbf{B}_{x x}^{(3)^{\mathrm{T}}}}{\partial \mathbf{u}_{\mathrm{L}}^{\mathrm{T}}} \varepsilon_{x x}^{(3)}+\frac{\partial \mathbf{B}_{x x}^{(4)}}{\partial \mathbf{u}_{\mathrm{L}}^{\mathrm{T}}} \varepsilon_{x x}^{(5)}+\frac{\partial \mathbf{B}_{x x}^{(5)^{\mathrm{T}}}}{\partial \mathbf{u}_{\mathrm{L}}^{\mathrm{T}}} \varepsilon_{x x}^{(4)}\right) \mathrm{d} x \\
& +\operatorname{EA} \int_{\mathrm{L}} \frac{\partial \mathbf{B}_{x x}^{(0)^{\mathrm{T}}}}{\partial \mathbf{u}_{\mathrm{L}}^{\mathrm{T}}} \mathbf{P} \mathrm{d} x \boldsymbol{\alpha}+\mathrm{EA}^{2}\left(\int_{\mathrm{L}} \mathbf{B}_{x x}^{(0)^{\mathrm{T}}} \mathbf{P} \mathrm{d} x\right) \mathbf{H}^{-1}\left(\int_{\mathrm{L}} \mathbf{P}^{\mathrm{T}} \mathbf{B}_{x x}^{(0)} \mathrm{d} x\right) \\
& +\mathrm{k}_{0} \mathrm{G}\left[\mathrm{I}_{y} \int_{\mathrm{L}}\left(\mathbf{B}_{x y}^{(2)}{ }^{\mathrm{T}} \mathbf{B}_{x y}^{(2)}+\frac{\left.\partial \mathbf{B}_{x y}^{(2)}\right)^{\mathrm{T}}}{\partial \mathbf{u}_{\mathrm{L}}^{\mathrm{T}}} \gamma_{x y}^{(2)}\right) \mathrm{d} x+\mathrm{A}^{2}\left(\int_{\mathrm{L}} \mathbf{B}_{x y}^{(0)^{\mathrm{T}}} \mathbf{P} \mathrm{d} x\right) \mathbf{H}^{-1} \int_{\mathrm{L}} \mathbf{P}^{\mathrm{T}} \mathbf{B}_{x y}^{(0)} \mathrm{d} x\right] \\
& +\mathrm{k}_{0} \mathrm{G}\left[\mathrm{I}_{z} \int_{\mathrm{L}}\left(\mathbf{B}_{x y}^{(1)^{\mathrm{T}}} \mathbf{B}_{x y}^{(1)}+\frac{\partial \mathbf{B}_{x y}^{(1)^{\mathrm{T}}}}{\partial \mathbf{u}_{\mathrm{L}}^{\mathrm{T}}} \gamma_{x y}^{(1)}+\mathbf{B}_{x z}^{(1)^{\mathrm{T}}} \mathbf{B}_{x z}^{(1)}+\frac{\partial \mathbf{B}_{x z}^{(1)}}{\partial \mathbf{u}_{\mathrm{L}}^{\mathrm{T}}} \gamma_{x z}^{(1)}\right) \mathrm{d} x\right] \\
& +\mathrm{k}_{0} \mathrm{GA}\left(\int_{\mathrm{L}} \frac{\partial \mathbf{B}_{x y}^{(0)^{\mathrm{T}}}}{\partial \mathbf{u}_{\mathrm{L}}^{\mathrm{T}}} \mathbf{P} \mathrm{d} x \boldsymbol{\beta}+\int_{\mathrm{L}} \frac{\partial \mathbf{B}_{x z}^{(0)^{\mathrm{T}}}}{\partial \mathbf{u}_{\mathrm{L}}^{\mathrm{T}}} \mathbf{P} \mathrm{d} x \boldsymbol{\chi}\right) \\
& +\mathrm{k}_{0} \mathrm{G}\left[\mathrm{I}_{y} \int_{\mathrm{L}}\left(\mathbf{B}_{x z}^{(2)^{\mathrm{T}}} \mathbf{B}_{x z}^{(2)}+\frac{\partial \mathbf{B}_{x z}^{(2)}}{\partial \mathbf{u}_{\mathrm{L}}^{\mathrm{T}}} \gamma_{x z}^{(2)}\right) \mathrm{d} x+\mathrm{A}^{2}\left(\int_{\mathrm{L}} \mathbf{B}_{x z}^{(0)^{\mathrm{T}}} \mathbf{P} \mathrm{d} x\right) \mathbf{H}^{-1} \int_{\mathrm{L}} \mathbf{P}^{\mathrm{T}} \mathbf{B}_{x z}^{(0)} \mathrm{d} x\right]
\end{aligned}
$$

where, $\mathbf{k}_{\mathrm{T}}$ is the sum of symmetric matrices, and some general matrices plus their transposes, thus $\mathbf{k}_{\mathrm{T}}$ is symmetric.

Gaussian integral procedure is adopted to calculate the internal force vector and tangent stiffness matrix,

$$
\begin{aligned}
\mathbf{f}= & \mathrm{E} \sum_{i=1}^{n_{0}}\left\{\left[\mathrm{I}_{y}\left(\mathbf{B}_{x x}^{(0)^{\mathrm{T}}} \varepsilon_{x x}^{(5)}+\mathbf{B}_{x x}^{(2)^{\mathrm{T}}} \varepsilon_{x x}^{(2)}+\mathbf{B}_{x x}^{(5)^{\mathrm{T}}} \varepsilon_{x x}^{(0)}\right)+\mathrm{I}_{\omega y} \mathbf{B}_{x x}^{(5)^{\mathrm{T}}} \varepsilon_{x x}^{(5)}\right] w_{\mathrm{T}}(i) \mathrm{J}\right\}_{\xi_{i}}+\mathrm{EA} \sum_{i=1}^{n_{0}}\left[\left(\mathbf{B}_{x x}^{(0)} \mathbf{P}^{\mathrm{T}}\right) w_{\mathrm{T}}(i) \mathrm{J}\right]_{\xi_{i}} \boldsymbol{\alpha} \\
& +\mathrm{E} \sum_{i=1}^{n_{0}}\left\{\left[\mathrm{I}_{z}\left(\mathbf{B}_{x x}^{(0)^{\mathrm{T}}} \varepsilon_{x x}^{(4)}+\mathbf{B}_{x x}^{(1)^{\mathrm{T}}} \varepsilon_{x x}^{(1)}+\mathbf{B}_{x x}^{(4)^{\mathrm{T}}} \varepsilon_{x x}^{(0)}\right)+\mathrm{I}_{\omega z} \mathbf{B}_{x x}^{(4)^{\mathrm{T}}} \varepsilon_{x x}^{(4)}\right] w_{\mathrm{T}}(i) \mathrm{J}\right\}_{\xi_{i}} \\
& +\mathrm{E} \mathrm{I}_{\omega y z} \sum_{i=1}^{n_{0}}\left[\left(\mathbf{B}_{x x}^{(4)^{\mathrm{T}}} \varepsilon_{x x}^{(5)}+\mathbf{B}_{x x}^{(5)^{\mathrm{T}}} \varepsilon_{x x}^{(4)}+\mathbf{B}_{x x}^{(3)^{\mathrm{T}}} \varepsilon_{x x}^{(3)}\right) w_{\mathrm{T}}(i) \mathrm{J}\right]_{\xi_{i}} \\
& +\mathrm{k}_{0} \mathrm{G}\left\{\sum_{i=1}^{n_{0}}\left[\left(\mathrm{I}_{y} \mathbf{B}_{x y}^{(2)^{\mathrm{T}}} \gamma_{x y}^{(2)}+\mathrm{I}_{z} \mathbf{B}_{x y}^{(1)^{\mathrm{T}}} \gamma_{x y}^{(1)}\right) w_{\mathrm{T}}(i) \mathrm{J}\right]_{\xi_{i}}+\mathrm{A} \sum_{i=1}^{n_{0}}\left(\mathbf{B}_{x y}^{(0)^{\mathrm{T}}} \mathbf{P} w_{\mathrm{T}}(i) \mathrm{J}\right)_{\xi_{i}} \boldsymbol{\beta}\right\} \\
& +\mathrm{k}_{0} \mathrm{G}\left\{\sum_{i=1}^{n_{0}}\left[\left(\mathrm{I}_{y} \mathbf{B}_{x z}^{(2)^{\mathrm{T}}} \gamma_{x z}^{(2)}+\mathrm{I}_{z} \mathbf{B}_{x z}^{(1)^{\mathrm{T}}} \gamma_{x z}^{(1)}\right) w_{\mathrm{T}}(i) \mathrm{J}\right]_{\xi_{i}}+\mathrm{A} \sum_{i=1}^{n_{0}}\left(\mathbf{B}_{x z}^{(0)^{\mathrm{T}}} \mathbf{P} w_{\mathrm{T}}(i) \mathrm{J}\right)_{\xi_{i}} \boldsymbol{\chi}\right\}
\end{aligned}
$$




$$
\begin{aligned}
& \mathbf{k}_{\mathrm{T}}=\mathrm{E} \sum_{i=1}^{n_{0}}\left\{\left[\mathrm{I}_{y}\left(\mathbf{B}_{x x}^{(2)^{\mathrm{T}}} \mathbf{B}_{x x}^{(2)}+\mathbf{B}_{x x}^{(5)^{\mathrm{T}}} \mathbf{B}_{x x}^{(0)}+\mathbf{B}_{x x}^{(0)} \mathbf{B}_{x x}^{\mathrm{T}} \mathbf{B}^{(5)}\right)+\mathrm{I}_{\omega y} \mathbf{B}_{x x}^{(5)}{ }^{\mathrm{T}} \mathbf{B}_{x x}^{(5)}\right] \mathcal{w}_{\mathrm{T}}(i) \mathrm{J}\right\}_{\xi_{i}} \\
& +\mathrm{E} \sum_{i=1}^{n_{0}}\left\{\left[\mathrm{I}_{z}\left(\mathbf{B}_{x x}^{(1)}\right)^{\mathrm{T}} \mathbf{B}_{x x}^{(1)}+\mathbf{B}_{x x}^{(4)}{ }^{\mathrm{T}} \mathbf{B}_{x x}^{(0)}+\mathbf{B}_{x x}^{(0)^{\mathrm{T}}} \mathbf{B}_{x x}^{(4)}\right)+\mathrm{I}_{\omega z} \mathbf{B}_{x x}^{(4)}{ }^{\mathrm{T}} \mathbf{B}_{x x}^{(4)}\right] \mathcal{w}_{\mathrm{T}}(i) \mathrm{J} \xi_{\xi_{i}} \\
& +\mathrm{EI}_{\omega y z} \sum_{i=1}^{n_{0}}\left[\left(\mathbf{B}_{x x}^{(3)^{\mathrm{T}}} \mathbf{B}_{x x}^{(3)}+\mathbf{B}_{x x}^{(4)^{\mathrm{T}}} \mathbf{B}_{x x}^{(5)}+\mathbf{B}_{x x}^{(5)}{ }^{\mathrm{T}} \mathbf{B}_{x x}^{(4)}\right) \mathcal{w}_{\mathrm{T}}(i) \mathrm{J}\right]_{\xi_{i}} \\
& +\mathrm{E} \sum_{i=1}^{n_{0}}\left\{\left[\mathrm{I}_{y}\left(\frac{\partial \mathbf{B}_{x x}^{(2)^{\mathrm{T}}}}{\partial \mathbf{u}_{\mathrm{L}}^{\mathrm{T}}} \varepsilon_{x x}^{(2)}+\frac{\partial \mathbf{B}_{x x}^{(5)}}{\partial \mathbf{u}_{\mathrm{L}}^{\mathrm{T}}} \boldsymbol{\varepsilon}_{x x}^{(0)}+\frac{\partial \mathbf{B}_{x x}^{(0)^{\mathrm{T}}}}{\partial \mathbf{u}_{\mathrm{L}}^{\mathrm{T}}} \boldsymbol{\varepsilon}_{x x}^{(5)}\right)+\mathrm{I}_{\omega y} \frac{\partial \mathbf{B}_{x x}^{(5)^{\mathrm{T}}}}{\partial \mathbf{u}_{\mathrm{L}}^{\mathrm{T}}} \boldsymbol{\varepsilon}_{x x}^{(5)}\right] w_{\mathrm{T}}(i) \mathrm{J}\right\}_{\xi_{i}} \\
& +\mathrm{E} \sum_{i=1}^{n_{0}}\left\{\left[\mathrm{I}_{z}\left(\frac{\partial \mathbf{B}_{x x}^{(1)}}{\partial \mathbf{u}_{\mathrm{L}}^{\mathrm{T}}} \varepsilon_{x x}^{(1)}+\frac{\partial \mathbf{B}_{x x}^{(4)^{\mathrm{T}}}}{\partial \mathbf{u}_{\mathrm{L}}^{\mathrm{T}}} \varepsilon_{x x}^{(0)}+\frac{\partial \mathbf{B}_{x x}^{(0)^{\mathrm{T}}}}{\partial \mathbf{u}_{\mathrm{L}}^{\mathrm{T}}} \varepsilon_{x x}^{(4)}\right)+\mathrm{I}_{\omega z} \frac{\partial \mathbf{B}_{x x}^{(4)}{ }^{\mathrm{T}}}{\partial \mathbf{u}_{\mathrm{L}}^{\mathrm{T}}} \varepsilon_{x x}^{(4)}\right] w_{\mathrm{T}}(i) \mathrm{J}\right\}_{\xi_{i}} \\
& +\mathrm{EI}_{\omega y z} \sum_{i=1}^{n_{0}}\left[\left(\frac{\partial \mathbf{B}_{x x}^{(3)}}{\partial \mathbf{u}_{\mathrm{L}}^{\mathrm{T}}} \varepsilon_{x x}^{(3)}+\frac{\partial \mathbf{B}_{x x}^{(4)^{\mathrm{T}}}}{\partial \mathbf{u}_{\mathrm{L}}^{\mathrm{T}}} \boldsymbol{\varepsilon}_{x x}^{(5)}+\frac{\partial \mathbf{B}_{x x}^{(5)^{\mathrm{T}}}}{\partial \mathbf{u}_{\mathrm{L}}^{\mathrm{T}}} \varepsilon_{x x}^{(4)}\right) w_{\mathrm{T}}(i) \mathrm{J}\right]_{\xi_{i}} \\
& +\mathrm{EA} \sum_{i=1}^{n_{0}}\left(\frac{\partial \mathbf{B}_{x x}^{(0)^{\mathrm{T}}}}{\partial \mathbf{u}_{\mathrm{L}}^{\mathrm{T}}} \mathbf{P} w_{\mathrm{T}}(i) \mathrm{J}\right)_{\xi_{i}} \boldsymbol{\alpha}+\mathrm{EA}^{2} \sum_{i=1}^{n_{0}}\left(\mathbf{B}_{x x}^{(0)}{ }^{\mathrm{T}} \mathbf{P} w_{\mathrm{T}}(i) \mathrm{J}\right)_{\xi_{i}} \mathbf{H}^{-1} \sum_{i=1}^{n_{0}}\left(\mathbf{P}^{\mathrm{T}} \mathbf{B}_{x x}^{(0)} w_{\mathrm{T}}(i) \mathrm{J}\right)_{\xi_{i}} \\
& +\mathrm{k}_{0} \mathrm{G}\left\{\mathrm{I}_{y} \sum_{i=1}^{n_{0}}\left[\left(\mathbf{B}_{x y}^{(2)} \mathbf{B}_{x y}^{(2)}+\frac{\partial \mathbf{B}_{x y}^{(2)}}{\partial \mathbf{u}_{\mathrm{L}}^{\mathrm{T}}} \gamma_{x y}^{(2)}\right) w_{\mathrm{T}}(i) \mathrm{J}\right]_{\xi_{i}}+\mathrm{A}^{2} \sum_{i=1}^{n_{0}}\left(\mathbf{B}_{x y}^{(0)^{\mathrm{T}}} \mathbf{P} w_{\mathrm{T}}(i) \mathrm{J}\right)_{\xi_{i}} \mathbf{H}^{-1} \sum_{i=1}^{n_{0}}\left(\mathbf{P}^{\mathrm{T}} \mathbf{B}_{x y}^{(0)} w_{\mathrm{T}}(i) \mathrm{J}\right)_{\xi_{i}}\right\} \\
& +\mathrm{k}_{0} \mathrm{GI}_{z} \sum_{i=1}^{n_{0}}\left[\left(\mathbf{B}_{x y}^{(1)^{\mathrm{T}}} \mathbf{B}_{x y}^{(1)}+\frac{\partial \mathbf{B}_{x y}^{(1)^{\mathrm{T}}}}{\partial \mathbf{u}_{\mathrm{L}}^{\mathrm{T}}} \gamma_{x y}^{(1)}+\mathbf{B}_{x z}^{(1)^{\mathrm{T}}} \mathbf{B}_{x z}^{(1)}+\frac{\partial \mathbf{B}_{x z}^{(1)}}{\partial \mathbf{u}_{\mathrm{L}}^{\mathrm{T}}} \gamma_{x z}^{(1)}\right) \mathcal{W}_{\mathrm{T}}(i) \mathrm{J}\right]_{\xi_{i}} \\
& +\mathrm{k}_{0} \mathrm{GA}\left[\sum_{i=1}^{n_{0}}\left(\frac{\partial \mathbf{B}_{x y}^{(0)^{\mathrm{T}}}}{\partial \mathbf{u}_{\mathrm{L}}^{\mathrm{T}}} \mathbf{P} w_{\mathrm{T}}(i) \mathrm{J}\right)_{\xi_{i}} \boldsymbol{\beta}+\sum_{i=1}^{n_{0}}\left(\frac{\partial \mathbf{B}_{x z}^{(0)}}{\partial \mathbf{u}_{\mathrm{L}}^{\mathrm{T}}} \mathbf{P} w_{\mathrm{T}}(i) \mathbf{J}\right)_{\xi_{i}} \boldsymbol{\chi}\right] \\
& +\mathrm{k}_{0} \mathrm{G}\left\{\mathrm{I}_{y} \sum_{i=1}^{n_{0}}\left[\left(\mathbf{B}_{x z}^{(2)^{\mathrm{T}}} \mathbf{B}_{x z}^{(2)}+\frac{\partial \mathbf{B}_{x z}^{(2)}}{\partial \mathbf{u}_{\mathrm{L}}^{\mathrm{T}}} \gamma_{x z}^{(2)}\right) w_{\mathrm{T}}(i) \mathrm{J}\right]_{\xi_{i}}+\mathrm{A}^{2} \sum_{i=1}^{n_{0}}\left(\mathbf{B}_{x z}^{(0)}{ }^{\mathrm{T}} \mathbf{P} w_{\mathrm{T}}(i) \mathrm{J}\right)_{\xi_{i}} \mathbf{H}^{-1} \sum_{i=1}^{n_{0}}\left(\mathbf{P}^{\mathrm{T}} \mathbf{B}_{x z}^{(0)} w_{\mathrm{T}}(i) \mathrm{J}\right)_{\xi_{i}}\right\}(30)
\end{aligned}
$$

where, $n_{0}$ is the number of Gaussian integral points along the central axis $\xi$ of element, $n_{0}=3$ in solving the examples below; $\xi_{i}$ and $w_{\mathrm{T}}(i)$ are the natural coordinate and weight factor at Gaussian point $i$, respectively; $\mathrm{J}$ is the Jacobian, $\mathrm{J}=\sum_{i=1}^{3} N_{i, \xi} x_{i 0} ; \boldsymbol{\alpha}, \boldsymbol{\beta}, \boldsymbol{\chi}$ can be calculated from Eqs. $26 \mathrm{a} \sim \mathrm{c}$, and

$$
\begin{aligned}
& \mathbf{H}=\mathrm{A} \sum_{i=1}^{n_{0}}\left[\mathbf{P}^{\mathrm{T}} \mathbf{P} w_{\mathrm{T}}(i) \mathrm{J}\right]_{\xi_{i}} \\
& \mathbf{F}_{1}=\mathrm{A} \sum_{i=1}^{n_{0}}\left[\mathbf{P}^{\mathrm{T}} \varepsilon_{x x}^{(0)} w_{\mathrm{T}}(i) \mathrm{J}\right]_{\xi_{i}}
\end{aligned}
$$




$$
\begin{aligned}
& \mathbf{F}_{2}=\mathrm{A} \sum_{i=1}^{n_{0}}\left[\mathbf{P}^{\mathrm{T}} \gamma_{x y}^{(0)} w_{\mathrm{T}}(i) \mathrm{J}\right]_{\xi_{i}} \\
& \mathbf{F}_{3}=\mathrm{A} \sum_{i=1}^{n_{0}}\left[\mathbf{P}^{\mathrm{T}} \gamma_{x z}^{(0)} w_{\mathrm{T}}(i) \mathrm{J}\right]_{\xi_{i}}
\end{aligned}
$$

The global internal force vector $\mathbf{f}_{\mathrm{G}}$ can be calculated from the local internal force vector $\mathbf{f}$,

$$
\mathbf{f}_{\mathbf{G}}=\mathbf{T}^{\mathrm{T}} \mathbf{f}
$$

where, $\mathbf{T}$ is the transformation matrix from the global coordinate system to the local coordinate system, it is calculated from

$$
\mathbf{T}=\frac{\partial \mathbf{u}_{\mathrm{L}}}{\partial \mathbf{u}_{\mathrm{G}}^{\mathrm{T}}}
$$

The global tangent stiffness matrix is derived from $\mathbf{f}_{\mathrm{G}}$ as below,

$$
\mathbf{k}_{\mathrm{TG}}=\frac{\partial \mathbf{f}_{\mathrm{G}}}{\partial \mathbf{u}_{\mathrm{G}}^{\mathrm{T}}}=\mathbf{T}^{\mathrm{T}} \frac{\partial \mathbf{f}}{\partial \mathbf{u}_{\mathrm{G}}^{\mathrm{T}}}+\frac{\partial \mathbf{T}^{\mathrm{T}}}{\partial \mathbf{u}_{\mathrm{G}}^{\mathrm{T}}} \mathbf{f}=\mathbf{T}^{\mathrm{T}} \mathbf{k}_{\mathrm{T}} \mathbf{T}+\frac{\partial \mathbf{T}^{\mathrm{T}}}{\partial \mathbf{u}_{\mathrm{G}}^{\mathrm{T}}} \mathbf{f}
$$

It is obvious that the first term in the right side of Eq. 37 is symmetric. The second term includes the second derivatives of local nodal variables with respect to global nodal variables, where the global nodal variables are commutative, thus the second term is also symmetric, resulting in a symmetric element tangent stiffness matrix $\mathbf{k}_{\mathrm{TG}}$ in the global coordinate system.

\section{CALCULATION OF EQUIVALENT EXTERNAL FORCE VECTOR}

In the present element formulation, vectorial rotational variables are employed to replace traditional angular rotational variables, thus the components of the internal force vector with respect to vectorial rotational variables are not moment and torque, and an equivalent external force vector must be adopted.

Firstly, assumed that Vector $\mathbf{e}_{n}$ is rotated through infinitesimal rotations of $\boldsymbol{\theta}^{\mathrm{T}}=\left\langle\begin{array}{lll}\delta \theta_{X} & \delta \theta_{Y} & \delta \theta_{Z}\end{array}\right.$ to become Vector $\mathbf{e}_{n+1}$, then an approximate relationship of $\mathbf{e}_{n}$ and $\mathbf{e}_{n+1}$ can be given as

$\mathbf{e}_{n+1}=[\mathbf{I}+\mathbf{S}(\delta \boldsymbol{\theta})] \mathbf{e}_{n}$

where, I is a $3 \times 3$ unit matrix, and

$\mathbf{S}(\delta \boldsymbol{\theta})=\left[\begin{array}{ccc}0 & -\delta \theta_{Z} & \delta \theta_{Y} \\ \delta \theta_{Z} & 0 & -\delta \theta_{X} \\ -\delta \theta_{Y} & \delta \theta_{X} & 0\end{array}\right]$ 
Eq. 38 can be rewritten as

$\mathbf{e}_{n+1}-\mathbf{e}_{n}=\mathbf{S}(\delta \boldsymbol{\theta}) \mathbf{e}_{n}=-\mathbf{S}\left(\mathbf{e}_{n}\right) \delta \boldsymbol{\theta}$

or

$\delta \mathbf{e}_{n}=-\mathbf{S}\left(\mathbf{e}_{n}\right) \delta \boldsymbol{\theta}$

thus the relationship between the principal vectors of the cross-section of the element at Node $i$ and the nodal angular rotations can be written as

$$
\left.\begin{array}{rl}
\delta \mathbf{e}_{i y} & =-\mathbf{S}\left(\mathbf{e}_{i y}\right) \delta \boldsymbol{\theta} \\
\delta \mathbf{e}_{i z} & =-\mathbf{S}\left(\mathbf{e}_{i z}\right) \delta \boldsymbol{\theta}
\end{array}\right\}
$$

furthermore, the relationship between the incremental vectorial rotational variables and the nodal angular rotational variables can be given as

$$
\left.\left\{\begin{array}{l}
\delta e_{i y, n_{i}} \\
\delta e_{i y, m_{i}} \\
\delta e_{i z, n_{i}}
\end{array}\right\}=-\left[\begin{array}{lll}
S_{n_{i}, 1}\left(\mathbf{e}_{i y}\right) & S_{n_{i}, 2}\left(\mathbf{e}_{i y}\right) & S_{n_{i}, 3}\left(\mathbf{e}_{i y}\right) \\
S_{m_{i}, 1}\left(\mathbf{e}_{i y}\right) & S_{m_{i}, 2}\left(\mathbf{e}_{i y}\right) & S_{m_{i}, 3}\left(\mathbf{e}_{i y}\right) \\
S_{n_{i}, 1}\left(\mathbf{e}_{i z}\right) & S_{n_{i}, 2}\left(\mathbf{e}_{i z}\right) & S_{n_{i}, 3}\left(\mathbf{e}_{i z}\right)
\end{array}\right]\right)\left\{\begin{array}{c}
\delta \theta_{i X} \\
\delta \theta_{i Y} \\
\delta \theta_{i Z}
\end{array}\right\}
$$

where, $S_{j, k}\left(\mathbf{e}_{i y}\right)$ and $S_{j, k}\left(\mathbf{e}_{i z}\right)$ are respectively the components of $\mathbf{S}\left(\mathbf{e}_{i y}\right)$ and $\mathbf{S}\left(\mathbf{e}_{i z}\right)$ at $\mathrm{j}^{\text {th }}$ row and $\mathrm{k}^{\text {th }}$ column.

In calculating the equivalent components of the external force vector with respect to vectorial rotational variables, the work done by the equivalent components must be equal to that done by the corresponding moment and torque at Node $i$,

$$
\left\{\begin{array}{l}
M_{e q 1} \\
M_{e q 2} \\
M_{e q 3}
\end{array}\right\}^{\mathrm{T}}\left\{\begin{array}{l}
\delta e_{i y, n_{i}} \\
\delta e_{i y, m_{i}} \\
\delta e_{i z, n_{i}}
\end{array}\right\}=\left\{\begin{array}{l}
M_{i X} \\
M_{i Y} \\
M_{i Z}
\end{array}\right\}^{\mathrm{T}}\left\{\begin{array}{l}
\delta \theta_{i X} \\
\delta \theta_{i Y} \\
\delta \theta_{i Z}
\end{array}\right\}
$$

where, $M_{\text {eqj }}(\mathrm{j}=1,2,3)$ is the equivalent component of the external force vector with respect to vectorial rotational variable, and $M_{i \alpha}(\alpha=X, Y, Z)$ is moment or torque loaded at Node $\mathrm{i}$.

Substitute Eq. 43 into Eq. 44, the equivalent components of the external force vector with respect to vectorial rotational variables can be calculated as

$$
\left\{\begin{array}{l}
M_{e q 1} \\
M_{e q 2} \\
M_{e q 3}
\end{array}\right\}=-\left[\begin{array}{lll}
S_{n_{i}, 1}\left(\mathbf{e}_{i y}\right) & S_{n_{i}, 2}\left(\mathbf{e}_{i y}\right) & S_{n_{i}, 3}\left(\mathbf{e}_{i y}\right) \\
S_{m_{i}, 1}\left(\mathbf{e}_{i y}\right) & S_{m_{i}, 2}\left(\mathbf{e}_{i y}\right) & S_{m_{i}, 3}\left(\mathbf{e}_{i y}\right) \\
S_{n_{i}, 1}\left(\mathbf{e}_{i z}\right) & S_{n_{i}, 2}\left(\mathbf{e}_{i z}\right) & S_{n_{i}, 3}\left(\mathbf{e}_{i z}\right)
\end{array}\right]^{-\mathrm{T}}\left\{\begin{array}{c}
M_{i X} \\
M_{i Y} \\
M_{i Z}
\end{array}\right\}
$$




\section{EXAMPLES}

\subsection{Locking Problem}

\subsubsection{Membrane locking problem}

An initially straight cantilever beam is subjected to an end bending moment (Figure 3). Its width and thickness are $\mathrm{b}$ and $\mathrm{h}$, respectively, the cross-sectional shear factor is $5 / 6$, and its length is $\mathrm{L}=100$; The material properties of the beam are $\mathrm{E}=2.1 \times 10^{7}$ and $\mu=0.3$, respectively.

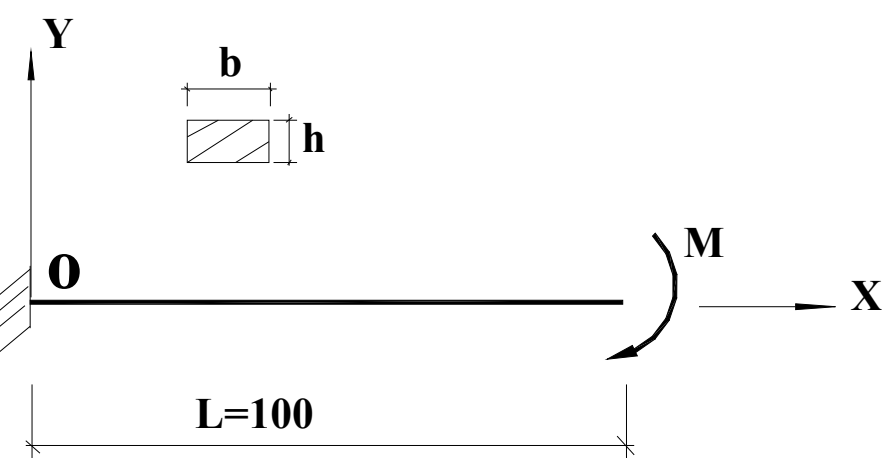

Figure 3. A Cantilever Beam subject to an End Bending Moment

Firstly, assumed that $\mathrm{b}=0.5$ and $\mathrm{h}=0.1$. The cantilever beam is divided into 7 elements equally. The deformed shapes of the cantilever at different end moment levels are depicted in Figure 4. It is shown that the beam experiences large displacement and large rotation, and its end rotation arrives at $2 \pi$ under $M=\frac{2 \pi E I}{L}$, the proposed beam element formulation demonstrates satisfying efficiency and reliability. Urthaler \& Reddy [13] and Lee [26] had also solved a similar problem, but they did not present the geometry and material properties of the cantilever beam.

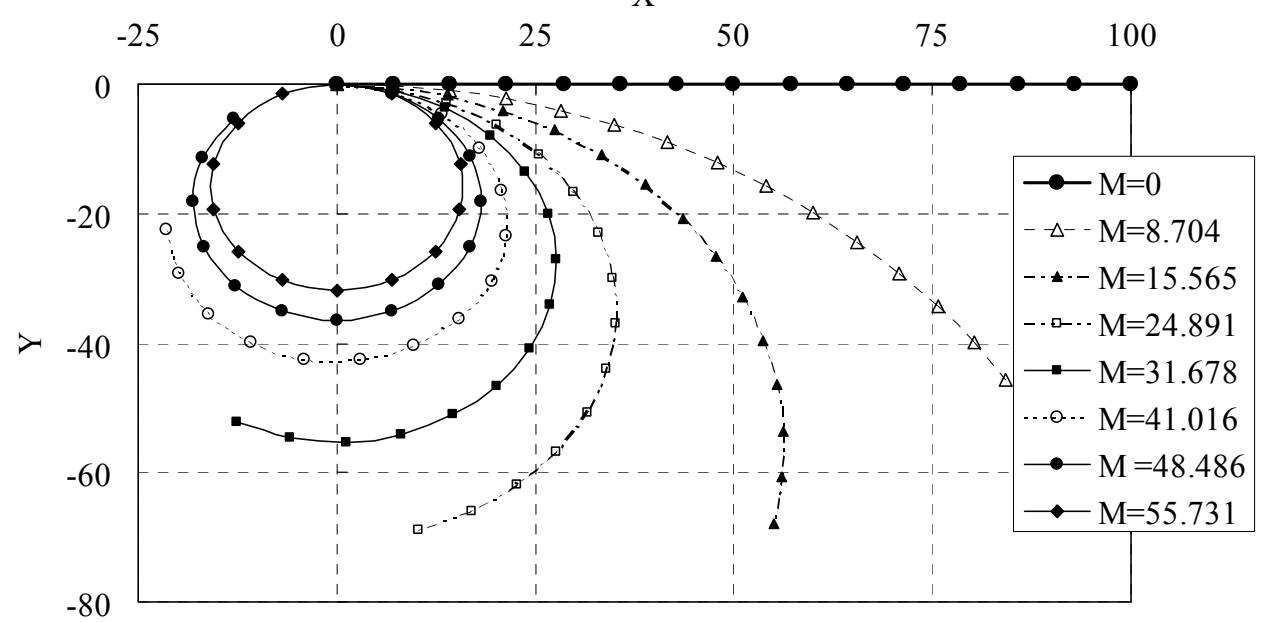

Figure 4. Deformed Shapes of the Cantilever Beam under Different End Moment Levels

To illuminate the computational efficiency and accuracy of the present beam element using assumed membrane strains and shear strains (for convenience, it is abbreviated as AM+AS element), 4 cantilever beams with the same width $(b=0.5)$ and different thickness values $(h=0.2,0.1$, $0.05,0.01)$ are solved respectively. For comparison, the theoretic solutions and the results from beam elements using conforming membrane strains and shear strains $(\mathrm{CM}+\mathrm{CS})$, conforming membrane strains and assumed shear strains $(\mathrm{CM}+\mathrm{AS})$, assumed membrane strains and conforming shear strains $(\mathrm{AM}+\mathrm{CS})$ are also given in Table 1. It is shown that numerical locking will become more serious in the $\mathrm{CM}+\mathrm{CS}$ and $\mathrm{CM}+\mathrm{AS}$ elements with the cantilever beam thickness decrease, 
and employing assumed shear strains or not has little effect on the computational efficiency and accuracy of these elements, however, adopting assumed membrane strains can eliminate numerical locking in the $\mathrm{AM}+\mathrm{CS}$ and $\mathrm{AM}+\mathrm{AS}$ elements effectively even if the cantilever beam thickness decreases greatly.

Table 1. End Moment Bending a Cantilever Beam into an Exact Complete Circle

\begin{tabular}{ccccc}
\hline Thickness h & 0.2 & 0.1 & 0.05 & 0.01 \\
\hline CM+CS-40e & $495.820(12.73 \%)$ & $82.988(50.95 \%)$ & $20.878(203.81 \%)$ & $2.856(--)$ \\
CM+AS-40e & $495.820(12.73 \%)$ & $82.982(50.94)$ & $20.878(203.81 \%)$ & $2.856(--)$ \\
AM+CS-7e & $445.913(1.38 \%)$ & $55.735(1.38)$ & $6.961(1.30 \%)$ & $5.574 \times 10^{-2}(1.38 \%)$ \\
AM+AS-7e & $445.909(1.38 \%)$ & $55.731(1.37 \%)$ & $6.968(1.40 \%)$ & $5.574 \times 10^{-2}(1.38 \%)$ \\
Exact values & 439.823 & 54.978 & 6.872 & $5.498 \times 10^{-2}$ \\
\hline
\end{tabular}

Note: "-40e" and "-7e" denote the element meshes employed; Values in the parentheses are the relative errors between the simulation results and the theoretical solutions.

\subsubsection{Shear locking problem}

A beam is clamped at both ends and loaded with a concentrated load at the central point (Figure 5a). It has a length of $2 \mathrm{~L}=20$, width $\mathrm{b}=0.5$ and thickness $\mathrm{h}$, and its material properties are $\mathrm{E}=2.1 \times 10^{7}$ and $\mu=0.3$, respectively. Considering the symmetry of its geometry and loading case, only one half of the beam is studied (Figure 5b).

(a)

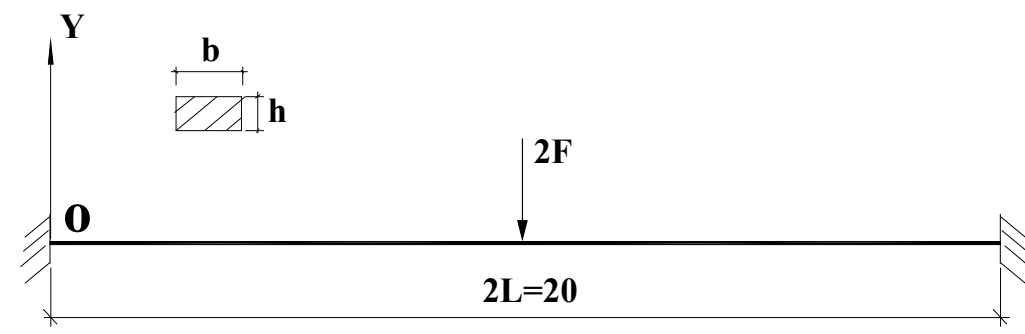

(b)

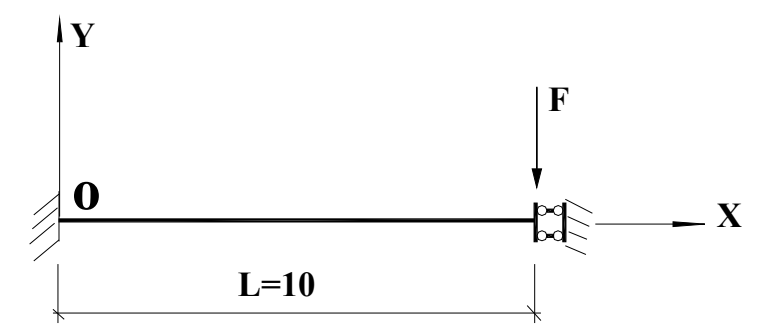

Figure 5. A Clamped Beam subject to a Concentrated Load at Central Point

Table 2. Deflection at the Loading Point of an End-Clamped Beam

\begin{tabular}{ccccc}
\hline Thickness h & 0.5 & 0.2 & 0.05 & 0.01 \\
\hline Load & 328.126 & 21.000 & $3.281 \times 10^{-1}$ & $2.625 \times 10^{-3}$ \\
\hline $\mathrm{CM}+\mathrm{CS}-2 \mathrm{e}$ & 0.1812 & 0.1407 & 0.0740 & 0.0283 \\
$-5 \mathrm{e}$ & 0.2173 & 0.1639 & 0.0829 & 0.0316 \\
$-10 \mathrm{e}$ & 0.2204 & 0.1667 & 0.0842 & 0.0323 \\
$\mathrm{CM}+\mathrm{AS}-2 \mathrm{e}$ & 0.2208 & 0.1673 & 0.0846 & 0.0326 \\
$-5 \mathrm{e}$ & 0.2208 & 0.1673 & 0.0846 & 0.0325 \\
$-10 \mathrm{e}$ & 0.2208 & 0.1673 & 0.0847 & 0.0325 \\
$\mathrm{AM}+\mathrm{CS}-2 \mathrm{e}$ & 0.1817 & 0.1418 & 0.0750 & 0.0288 \\
$-5 \mathrm{e}$ & 0.2173 & 0.1639 & 0.0829 & 0.0316 \\
$-10 \mathrm{e}$ & 0.2205 & 0.1667 & 0.0842 & 0.0323 \\
$\mathrm{AM}+\mathrm{AS}-2 \mathrm{e}$ & 0.2212 & 0.1678 & 0.0849 & 0.0326 \\
$-10 \mathrm{e}$ & 0.2208 & 0.1673 & 0.0847 & 0.0325 \\
\hline
\end{tabular}


Different beam thickness values $(\mathrm{h}=0.5,0.2,0.05$ and 0.01$)$ are considered. For comparison, the deflections at the loading point calculated by using AS+AM, CS+CM, AS+CM and CS+AM elements using different element meshes are presented in Table 2. It demonstrates that the convergence of the $\mathrm{CM}+\mathrm{CS}$ and $\mathrm{AM}+\mathrm{CS}$ elements become deteriorated with the beam thickness decrease, even assumed membrane strains are introduced in $\mathrm{AM}+\mathrm{CS}$ element, thus fine element mesh must be employed to get accurate solutions; while the thickness variation has little effect on the computational accuracy and convergence of the AM+AS and $\mathrm{CM}+\mathrm{AS}$ elements, satisfying solutions can be achieved by using very coarse element meshes of them.

\subsubsection{Membrane and shear locking problems}

A cantilever beam is subjected to a concentrated load at the free end (Figure 6). It has a length $\mathrm{L}=5$, width $b=0.5$ and thickness $h$; its material properties are $E=2.1 \times 10^{7}$ and $\mu=0.3$, respectively.

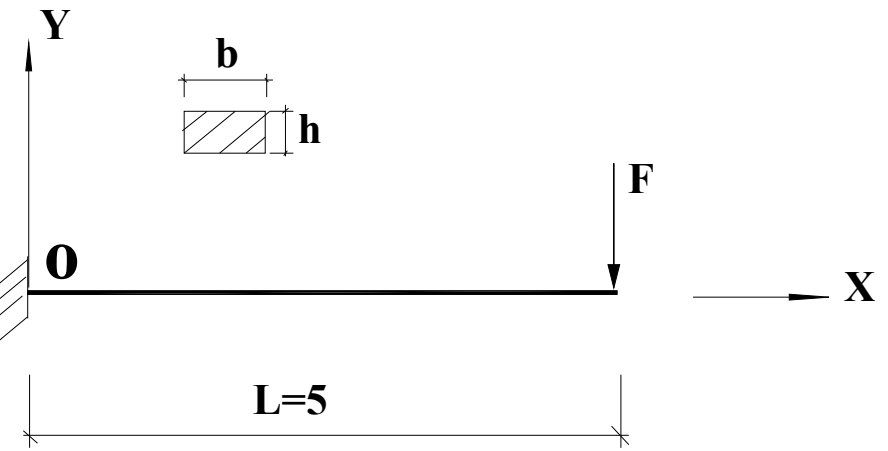

Figure 6. A Cantilever Beam subject to a Concentrated Load at Free End

Different thickness values of the cantilever beam are considered, and the results calculated by using $\mathrm{CM}+\mathrm{CS}, \mathrm{CM}+\mathrm{AS}, \mathrm{AM}+\mathrm{CS}$ and $\mathrm{AM}+\mathrm{AS}$ elements are presented in Table 3. It is shown that numerical locking occurs in $\mathrm{CM}+\mathrm{CS}, \mathrm{CM}+\mathrm{AS}$ and $\mathrm{AM}+\mathrm{CS}$ elements, it becomes more serious with beam thickness decrease, and this tendency is even more obvious in $\mathrm{CM}+\mathrm{CS}, \mathrm{CM}+\mathrm{AS}$ elements, while the AM+AS element is free of locking.

Table. 3 Deflection at the Free End of a Cantilever Beam

\begin{tabular}{rcccc}
\hline Thickness h & 0.5 & 0.2 & 0.05 & 0.01 \\
\hline Load & 656.250 & 42.000 & $6.563 \times 10^{-1}$ & $5.250 \times 10^{-3}$ \\
\hline CM+CS-1e & 0.1942 & 0.1725 & 0.1123 & 0.0472 \\
$-2 \mathrm{e}$ & 0.2412 & 0.2322 & 0.2003 & 0.1097 \\
$-10 \mathrm{e}$ & 0.2513 & 0.2495 & 0.2487 & 0.2461 \\
$\mathrm{CM}+\mathrm{AS}-1 \mathrm{e}$ & 0.2480 & 0.2332 & 0.1746 & 0.1097 \\
$-2 \mathrm{e}$ & 0.2508 & 0.2464 & 0.2158 & 0.1253 \\
$-10 \mathrm{e}$ & 0.2513 & 0.2497 & 0.2493 & 0.2467 \\
$\mathrm{AM}+\mathrm{CS}-1 \mathrm{e}$ & 0.1975 & 0.1890 & 0.1872 & 0.1872 \\
$-2 \mathrm{e}$ & 0.2417 & 0.2355 & 0.2339 & 0.2338 \\
$-10 \mathrm{e}$ & 0.2513 & 0.2495 & 0.2488 & 0.2487 \\
$\mathrm{AM}+\mathrm{AS}-1 \mathrm{e}$ & 0.2513 & 0.2496 & 0.2494 & 0.2493 \\
$-10 \mathrm{e}$ & 0.2513 & 0.2497 & 0.2494 & 0.2494
\end{tabular}

Based on the three examples above, several conclusions can be drawn: 1) membrane locking exists in the first example, it becomes even more serious in a thin beam element, introducing assumed membrane strains in a Hellinger-Reissner functional can exclude membrane locking effectively; 2) shear locking occurs in the second example, and assumed shear strains in a Hellinger-Reissner functional can eliminate it successfully; 3 ) both membrane locking and shear locking are observed 
in the third example, and employing assumed membrane strains and shear strains simultaneously can avoid them; 4) locking phenomena are closely related to element thickness, loading cases and boundary conditions, etc., and they may even occur in thick beam problems.

\subsection{A Cantilever $45^{\circ}$ - bend subject to End Loading}

A cantilever $45^{\circ}$ - bend lies in X-Y plane (see Figure 7), it has an average radius of 100in, and a square cross-section of $1 \times 1 \mathrm{in}^{2}$, its elastic modulus $\mathrm{E}$ and Poisson's ratio $\mu$ are $10^{7} \mathrm{psi}$ and 0.0 , respectively. A concentrated load in Z-direction is applied at the free end.

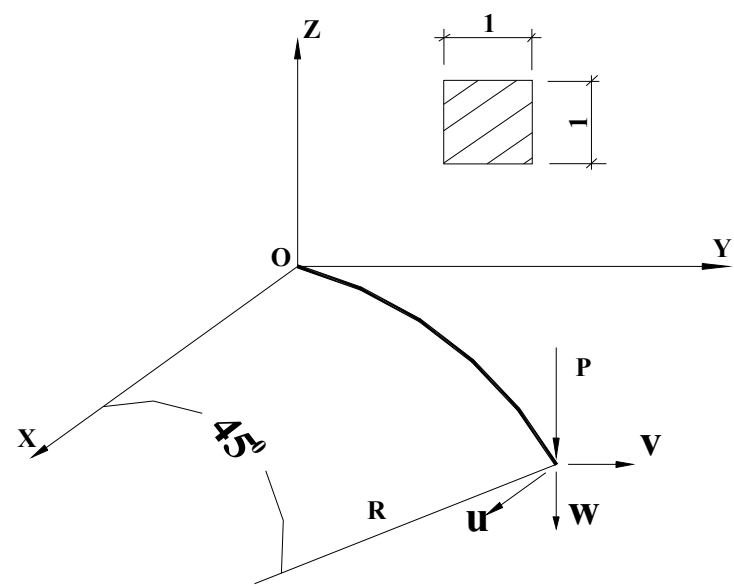

Figure 7. A Cantilever $45^{\circ}$-bend with a Concentrated Tip Load

This bend is divided into 8 beam elements equally, and these elements are idealized as straight beams. The tip displacements under different load levels are given in Table 4. To verify the reliability and accuracy of the procedure, the results from Bathe \& Bolourchi [27] and Simo \& Vu-Quoc [17] are also presented in Table 4, it is shown that the results from present studies can fit in well with them.

Table 4. Tip Displacements under Different Load Levels

\begin{tabular}{|c|c|c|c|c|c|c|c|c|c|}
\hline \multirow{3}{*}{$\begin{array}{l}\text { Load } \\
\text { level } \\
\text { (lb) }\end{array}$} & \multicolumn{9}{|c|}{ Tip displacement (in) } \\
\hline & \multicolumn{3}{|c|}{ Present study } & \multicolumn{3}{|c|}{ Bathe and Bolourchi [27] } & \multicolumn{3}{|c|}{ Simo and Vu-Quoc [17] } \\
\hline & $\mathrm{u}$ & $\mathrm{v}$ & $\mathrm{w}$ & $\mathrm{u}$ & $\mathrm{v}$ & W & $\mathrm{u}$ & $\mathrm{v}$ & $\mathrm{w}$ \\
\hline 300 & -7.20 & -12.21 & -40.53 & -6.8 & -11.5 & -39.5 & -6.97 & -11.86 & -40.08 \\
\hline 450 & -10.94 & -18.78 & -48.75 & -- & -- & -- & -10.68 & -18.38 & -48.39 \\
\hline 600 & -13.75 & -23.86 & -53.64 & -13.4 & -23.5 & -53.4 & -13.51 & -23.47 & -53.37 \\
\hline
\end{tabular}

\subsection{A Space Arc Frame Subject to Concentrated Loading}

This arc frame consists of two groups of members (Figure 8). The cross-section properties of the members in the arc frame planes are $\mathrm{A}_{1}=0.5, \mathrm{I}_{\mathrm{yl}}=0.4$ and $\mathrm{I}_{\mathrm{z} 1}=0.133$, respectively, and for the rib members, $\mathrm{A}_{2}=0.1, \mathrm{I}_{\mathrm{y} 2}=0.05$ and $\mathrm{I}_{\mathrm{z} 2}=0.05$, respectively. The material properties are $\mathrm{E}=4.32 \times 10^{5}$ and $\mathrm{G}=1.66 \times 10^{5}$. This frame is pinned at four boundary nodes. In addition to four vertical concentrated loads $\mathrm{P}$, the structure is also subjected to two lateral concentrated loads $0.001 \mathrm{P}$ (Figure 8). 


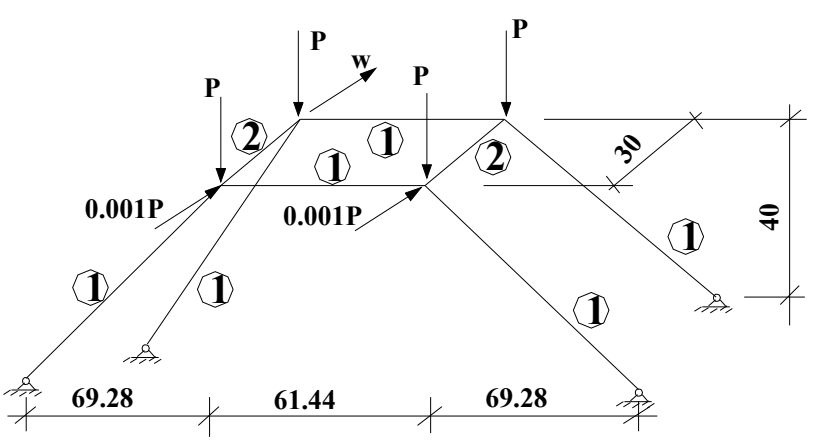

Figure 8. Space Arc Frame

In numerical analysis, each member is treated as one element. The deflection curve at Point A of the arc frame is presented in Figure 9, it is in close agreement with the solution from Wen and Rahimzadeh [28].

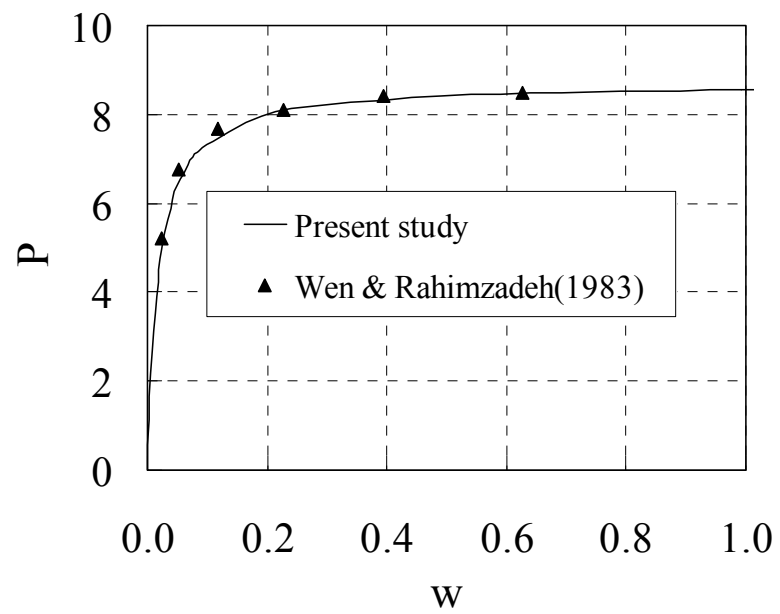

Figure 9. Response of Space Arc Frame under Ultimate Concentrated Loading

\section{CONCLUSIONS}

A mixed co-rotational 3D beam element formulation is proposed by using the Hellinger-Reissner functional, where vectorial rotational variables are employed to replace traditional angular rotational variables, taking advantages in calculating the element tangent stiffness matrix. The equivalent components of the external force vector with respect to vectorial rotational variables can be calculated directly from corresponding end moment and torque, thus this element can also be used in modelling of beams subject to end moment and torque. Through three patch tests of locking problems, the present beam element demonstrates its locking-free behaviours, and its computational accuracy and efficiency are verified by multiple elastic examples of large displacement and large rotation problems.

\section{ACKNOWLEDGEMENTS}

This work is supported by Qianjiang Program for Talented Oversea Returnees, Chinese Universities Scientific Fund and Aerospace Support Technology Fund. In addition, it also benefits from National Natural Science Foundation of China (50408022), the Future Academic Star Program of Zhejiang University, the financial supports of the Scientific Research Foundation for the Returned Overseas Chinese Scholars, provided respectively by State Education Ministry and Zhejiang Province. 


\section{REFERENCES}

[1] Rankin, C.C. and Brogan, F.A., "An Element Independent Corotational Procedure for the Treatment of Large Rotation", Journal of Pressure Vessel Technology-Transactions of The ASME, 1986, Vol. 108, No. 2, pp. 165-174.

[2] Crisfield, M.A., "Nonlinear Finite Element Analysis of Solid and Structures", John Wiley \& Sons, Chichester, 1996, Vol. 2.

[3] Yang, H.T.Y., Saigal, S., Masud, A. and Kapania, R.K., "Survey of Recent Shell Finite Elements", International Journal for Numerical Methods in Engineering, 2000, Vol. 47, No.1, pp. 101-127.

[4] Wempner, G., "Finite Elements, Finite Rotations and Small Strains of Flexible Shells", International Journal of Solids and Structures, 1969, Vol. 5, No. 2, pp. 117-153.

[5] Belytschko, T. and Hseih, B.J., "Non-linear Transient Finite Element Analysis with Convected Co-ordinates", International Journal for Numerical Methods in Engineering, 1973, Vol. 7, No. 3, pp. 255-271.

[6] Belytschko, T. and Glaum, L.W., "Application of Higher Order Corotational Stretch Theories to Nonlinear Finite Element Analysis", Computers \& Structures, 1979, Vol. 10, No. 1-2, pp. 175-182.

[7] Argyris, J.H., Bahner, H., Doltsnis, J., et al., "Finite Element Method - the Natural Approach", Computer Methods in Applied Mechanics and Engineering, 1978, Vol.17/18, Part 1, pp. 1-106.

[8] Oran, C., "Tangent Stiffness in Plane Frames", Journal of the Structural Division, ASCE, 1973, Vol. 99, ST6, pp. 973-985.

[9] Oran, C., "Tangent Stiffness in Space Frames", Journal of the Structural Division, ASCE, 1973, Vol. 99, ST6, pp. 987-1001.

[10] Stolarski, H., Belytschko, T. and Lee, S.H., "Review of Shell Finite Elements and Corotational Theories", Computational Mechanics Advances, 1995, Vol. 2, No. 2, pp. 125-212.

[11] Crisfield, M.A. and Moita, G.F., "A Unified Co-rotational Framework for Solids, Shells and Beams", International Journal of Solids and Structures, 1996, Vol. 33, No. 20-22, pp. 2969-2992.

[12] Felippa, C.A. and Haugen, B., "A Unified Formulation of Small-strain Corotational Finite Elements, I. Theory", Computer Methods in Applied Mechanics and Engineering, 2005, Vol. 194, No. 21-24, pp. 2285-2335.

[13] Urthaler, Y. and Reddy, J.N., "A Corotational Finite Element Formulation for the Analysis of Planar Beams", Communications in Numerical Methods in Engineering, 2005, Vol. 21, No. 10, pp. 553-570.

[14] Galvanetto, U. and Crisfield, M.A., "An Energy-conserving Co-rotational Procedure for the Dynamics of Planar Beam Structures", International Journal for Numerical Methods in Engineering, 1996, Vol. 39, No. 13, pp. 2265-2282.

[15] Iura, M., Suetake, Y. and Atluri, S.N., "Accuracy of Co-rotational Formulation for 3-D Timoshenko's Beam”, CMES-Computer Modeling In Engineering \& Sciences, 2003, Vol. 4, No. 2, pp. 249-258.

[16] Pajot, J.M. and Maute, K., "Analytical Sensitivity Analysis of Geometrically Nonlinear Structures Based on the Co-rotational Finite Element Method", Finite Elements in Analysis and Design, 2006, Vol. 42, No. 10, pp. 900-913.

[17] Simo, J.C. and Vu-Quoc, L., "A Three-dimensional Finite-strain Rod Model. Part II, Computational Aspects", Computer Methods in Applied Mechanics and Engineering, 1986, Vol. 58, No. 1, pp. 79-116. 
[18] Jelenic, G. and Crisfield, M.A., "Problems Associated with the Use of Cayley Transform and Tangent Scaling for Conserving Energy and Momenta in the Reissner-Simo Beam Theory", Communications in Numerical Methods in Engineering, 2002, Vol. 18, No. 10, pp.711-720.

[19] McRobie, F.A. and Lasenby, J., "Simo-Vu Quoc rods using Clifford algebra", International Journal for Numerical Methods in Engineering, 1999, Vol. 45, No. 4, pp. 377-398.

[20] Crisfield, M.A., "Consistent Co-rotational Formulation for Non-linear, Three-dimensional, Beam-elements", Computer Methods in Applied Mechanics and Engineering, 1990, Vol. 81, No. 2, pp. 131-150.

[21] Simo, J.C., "(Symmetric) Hessian for Geometrically Nonlinear Models in Solid Mechanics, Intrinsic Definition and Geometric Interpretation", Computer Methods in Applied Mechanics and Engineering, 1992, Vol. 96, No. 2, pp. 189-200.

[22] Li, Z.X., "A Mixed Co-rotational Formulation of 2D Beam Element Using Vectorial Rotational Variables", Communications in Numerical Methods in Engineering, 2007, Vol. 23, No. 1, pp. 45-69.

[23] Li, Z.X., "A Co-rotational Formulation for 3D Beam Element Using Vectorial Rotational Variables”, Computational Mechanics, 2007, Vol. 39, No. 3, pp. 293-308.

[24] Li, Z.X. and Vu-Quoc, L., "An Efficient Co-rotational Formulation for Curved Triangular Shell Element", International Journal for Numerical Methods in Engineering, 2007,Vol.72, No. 9, pp. 1029-1062.

[25] Li, Z.X., Izzuddin, B.A. and Vu-Quoc, L., "A 9-node Co-rotational Quadrilateral Shell Element”, Computational Mechanics, 2008, Vol. 42, No. 6, pp. 873-884.

[26] Lee, K., "Analysis of Large Displacements and Large Rotations of Three-dimensional Beams by Using Small Strains and Unit Vectors", Communications in Numerical Methods in Engineering, 1997, Vol. 13, No. 12, pp. 987-997.

[27] Bathe, K.J. and Bolourchi, S., "Large Displacement Analysis of Three-dimensional Beam Structures", International Journal for Numerical Methods in Engineering, 1979, Vol. 14, No.7, pp. 961-986.

[28] Wen, R.K. and Rahimzadeh, J., "Nonlinear Elastic Frame Analysis by Finite Element", Journal of the Structural Division, ASCE, 1983, Vol. 109, No. 8, pp. 1951-1971. 